\title{
LUTTE INTERNATIONALE CONTRE LE TERRORISME, SÉCURITÉ INTERNATIONALE ET DROITS FONDAMENTAUX. LES PIROUETTES DES JUGES EUROPÉENS ENTRE CREATION DE HIÉRARCHIES NORMATIVES IMPROBABLES ET SACRIFICE DES GARANTIES JURIDICTIONNELLES LES PLUS ÉLEMENTAIRES*
}

\begin{abstract}
Alberto PUPPO**
RESUMEN: La preocupación esencial de la comunidad internacional parece ser protegerse contra el riesgo "terrorista", libre de poner entre paréntesis al Estado de derecho. Las organizaciones internacionales, por medio de las instituciones europeas, deciden ahora quiénes son "buenos ciudadanos" y quiénes no lo son. Las sentencias Yusuf, Bosphorus y Behrami, dictadas de los tribunales europeos, parecen confirmar el estatuto particular de la cooperación internacional: la invocación del capítulo VII de la Carta de la ONU podría funcionar como una cláusula de licitud. El juez comunitario parece salirse de esta situación incómoda construyendo improbables jerarquías normativas.
\end{abstract}

ABSTRACT: The main preoccupation of the international community seems to be to protect itself against the "terrorist" risk, even if it means to put between parentheses the Rule of Law. The international organizations, with the collaboration of the European institutions, now decide who are "good citizens" and who are not. The judgments Yusuf, Bosphorus and Behrami, of the European Courts, seem to confirm the particular status of the international cooperation: the invocation of chapter VII of the UN Charter risk to make lawful any violation of human rights. The communitarian judge seems to get out of this embarrassing situation by constructing improbable normative hierarchies.

RÉSUMÉ: La préoccupation essentielle de la communauté internationale semble être de se protéger contre le risque "terroriste", quitte à mettre entre parenthèses l'Etat de droit. Les organisations internationales, par l'intermédiaire des institutions européennes, décident désormais qui est "bon citoyen" et qui ne l'est pas. Les arrêts Yusuf, Bosphorus et Behrami, rendus par les juridictions européennes, semblent confirmer le statut particulier de la coopération internationale: l'invocation du chapitre VII de la Charte de l'ONU risque de fonctionner comme une clause de licéité. Le juge communautaire semble se sortir de cette situation embarrassante en construisant d'improbables hiérarchies normatives.

* Artículo recibido el 10 de julio de 2008 y aceptado para su publicación el 4 de agosto de 2008.

** Doctor en filosofía analítica y teoría general del derecho (Milán), investigador del Centre de Théorie et d'Analyse du Droit (París), y profesor de Derecho comunitario y de Teoría del derecho internacional. 
SOMMAIRE: I. Introduction. II. Le phénomène des listes noires ou de l'effet redoutable de la coopération internationale. III. Bosphorus, Yusuf, Berhami: ou de l'effet "Blanchissant" du chapitre VII de la Charte de l'ONU et de la coopération internationale. IV. Conclusion: vers un retour à l'Etat de droit?

\section{INTRODUCTION}

Le droit international, en tant que production normative "molle" et, pour une partie importante, de nature coutumière, a indirectement fait l'objet des travaux d'Herbert Hart: ${ }^{1}$ dans quelle mesure le droit international peut être considéré comme étant un "droit" à part entière? La tradition positiviste classique ayant souvent abouti à la thèse de la nature purement morale et/ou politique des obligations "internationales", Hart a construit son concept de droit à partir de la notion d'acceptation, ${ }^{2}$ et non de la notion de sanction. Or, si le droit international a été longtemps dépourvu de sanctions, on ne peut pas nier l'existence de normes coutumières dont l'émergence repose sur l'acceptation, de la part des Etats (à la fois créateurs et destinataires de ces normes), du caractère "obligatoire" de certains comportements.

Plus la règle s'éloigne du modèle de la règle de conduite que l'on retrouve dans la plupart des sociétés, plus la catégorie des acteurs de l'acceptation tend à coïncider avec celle des professionnels du droit, notamment les juges. Or, les juridictions internationales se multiplient et leur rôle devient de plus en plus celui de (re)tracer les frontières entre les différents systèmes juridiques (nationaux, régionaux et internationaux), voire de les hiérarchiser. La protection internationale des droits de l'homme et la poursuite internationale, par exemple, des auteurs (ou financeurs) d'actes terroristes, impose en effet de repenser, à la fois le rapport liant l'individu à l'Etat et le rapport liant l'Etat à la communauté internationale. Un nouveau paysage se dessine: dans ce dessin une nouvelle figure,

Note: La Cour de justice a suivi les conclusions de l'Avocat général et annulé l'arrêt Kadi, ainsi que le règlement communautaire, par une décision du 3 septembre 2008.

1 Surtout dans son ouvrage The Concept of Law, London, Oxford University Press, 1961.

2 Puppo, A., L'acceptation dans le droit. Eléments pour une théorie générale, à paraître. 
celle de l'individu en tant que "sujet" en droit international, semble trouver ses contours. ${ }^{3}$

Les évolutions récentes de l'action du Conseil de Sécurité de l'ONU, et les prises de positions des juges européens, la Cour Européenne des Droits de l'Homme (CEDH) ${ }^{4}$ et le Tribunal de Première Instance des Communautés Européennes (TPICE) $)^{5}$ en matière de sanctions économiques décidées par l'ONU et touchant des individus et/ou des entités privées, imposent un questionnement. Quelle est la nouvelle place que l'individu occupe dans un système juridique internationalisé, projeté dans une lutte contre un ennemi déterritorialisé ${ }^{6}$ — mais connoté culturellement-, le terrorisme?

L'affaire Yusuf, tranchée par le TPICE, ${ }^{7}$ est l'histoire d'un citoyen suédois dont le nom, au lendemain des attentats du 11 septembre 2001, est inscrit sur une liste de personnes (supposées être) ${ }^{8}$ liées au réseau Al-Quaida. L'action combinée des Nations Unies (par la biais du Comité des sanctions), de l'Union européenne (par l'adoption d'un règlement transcrivant la liste de noms établie par le Comité des sanctions), et des Etats (liés en vertu du principe de primauté du droit communautaire),

3 Voir sur cette question, Société française de droit international, Le sujet en droit international, Paris, Pédone, 2005, notamment la contribution de Florent, J. L., "Les destinataires non étatiques des résolutions du Conseil de sécurité”, pp. 107-117.

4 CEDH, Arrêt de Grande Chambre du 30 juin 2005, Bosphorus Airways c. Irlande; CEDH, Grande chambre, décision sur la recevabilité du 31 mai 2007, Behrami c. France et Saramati c. la France, l'Allemagne et la Norvège.

5 TPICE, 21 septembre 2005, affaires jointes T-306/01 Ahmed Ali Yusuf et Al Barakaat International Foundation c. Conseil de l'Union europénne, et T-315/01, Yassin Abdullah Kadi c. Conseil de l'Union europénne. Voir aussi: TPICE, 12 juillet 2006, affaires jointes T-253/02 Chafiq Ayadi Kadi c. Conseil de l'Union européenne, et T-49/04 Faraj Hassan c. Conseil de l'Union européenne et Commission des Communautéseuropéennes.

6 En effet, comme le souligne J. L. Florent, op. cit., note 3, p. 110, à propos de la lutte contre le terrorisme telle que mise en place après le 11 septembre 2001, "ce régime de sanctions... est d'autant plus remarquable qu'il est entièrement déterritorialisé".

7 TPICE, Yusuf, op. cit., note 5.

8 Sur le rôle du gouvernement américain dans l'établissement de ces listes et, plus en général, sur le fonctionnement du Comité, voir Rosand, E., "The security council's efforts to monitor the implementation of al qaeda/taliban sanctions", AJIL, 2004, vol. 98, p. 744 . 
aboutit à l'application de sanctions économiques très lourdes à son encontre tout en le privant de tout moyen de recours effectif. ${ }^{9}$

La préoccupation essentielle de la communauté internationale semble celle de se protéger par tout moyen contre le risque "terroriste", quitte à mettre entre parenthèses ce qui peut être considéré, quoi que l'on pense de son présupposé idéologique, un acquis des Etats contemporains, à savoir la garantie des droits fondamentaux. Les organisations internationales, par l'intermédiaire des institutions européennes, décident désormais qui est "bon citoyen" et qui ne l'est pas. L'arrêt Bosphorus, rendu par la $\mathrm{CEDH},{ }^{10}$ semble confirmer le statut particulier de la coopération internationale, notamment en matière de maintien de la paix et de la sécurité internationales: en tant qu'intérêt supérieur blanchissant, pour ainsi dire, toute mesure portant atteinte aux droits fondamentaux garantis par la convention. L'invocation des obligations découlant de la coopération internationale, en particulier celles découlant de la Charte de l'ONU, fonctionne alors comme une clause de licéité. ${ }^{11}$

Un tel constat, nourri par la récente jurisprudence européenne en matière de sanctions économiques individuelles, invite à (re)poser la question des rapports entre le système juridique européen et l'ordre juridique international, question à laquelle le Tribunal de Première Instance des Communautés Européenne semble avoir donné une curieuse réponse. Il faut préciser que, si l'application de sanctions économiques par l'ONU, telles des mesures d'embargo, n'était pas une nouveauté, l'institution des comités de sanctions a marqué le début d'une nouvelle ère, ${ }^{12}$ dans laque-

9 Cet aspect semble désormais faire l'objet d'un constat généralisé. En ce sens, voir le rapport de Ian Cameron, The European Convention on Human Rights, Due Process and United Security Council Counter-Terrorism Sanctions, Conseil de l'Europe, 6 février 2006; et celui de Fassbender, Bardo, Targeted Sanctions and Due Process, Bureau des affaires juridiques des Nations-Unies, 20 mars 2006.

10 Arrêt du 30 juin 2005, op. cit., note 4.

11 Sur cet aspect de l'arrêt, voir au moins Ciampi, A., "L’Union européenne et le respect des droits de l'homme dans la mise en œuvre des sanctions devant la Cour européenne des droits de l'homme", RGDIP, p. 86. Voir aussi Cannizzaro, E., "Sulla responsabilità internazionale per condotte degli Stati membri dell'Unione europea: in margine al caso Bosphorus", Rivista di diritto internazionale (RDI), vol. 3, 2005, p. 762.

12 Il s'agit du Comité des sanctions créé, quatre jours après l'invasion du Koweit par l'Irak, par la résolution 661, du 6 août 1990, adoptée par 13 voix, avec aucune voix contre et 2 abstentions (Cuba et Yemen). Pour une étude approfondie de ce comité, voir Koskenniemi, M., "Le comité des sanctions (crée par la résolution 661 du Conseil de Sécuri- 
lle s'inscrit le comité créé pour lutter contre le financement du terrorisme et notamment du réseau d'Al-Quaida. ${ }^{13}$ Afin de bien comprendre une telle nouveauté, il ne faut pas oublier que le terrorisme et son financement font l'objet d'une convention internationale qui n'est pas encore en vigueur. Le Conseil de Sécurité, traditionnellement, se limitait à inviter les Etats à ratifier les conventions existantes. Or, l'urgence terroriste a changé cette pratique: cela est confirmé, comme le précise une fine et récente analyse de l'activité "législatrice" du Conseil de Sécurité, par le fait que la résolution 1373, dans sa première partie, reprend le contenu de la Convention Internationale pour la répression du financement du terrorisme de 1999. Ce qui traduit la volonté de rendre effectifs les engagements conventionnels sans attendre l'aboutissement du processus de ratification. ${ }^{14}$

L'activité de ce dernier comité des sanctions mérite une attention particulière pour plusieurs raisons: d'abord car ses sanctions concernent des individus et non plus des Etats, ensuite car ses décisions ont été retranscrites, selon des modalités qui suscitent de nombreuses perplexités, par l'Union européenne, et, enfin, car les effets de ces sanctions reposent avec acuité la question de la protection des droits fondamentaux de certaines personnes qui, en dépit du fait d'avoir la nationalité de l'un des Etats membres de l'Union sont considérées comme suspectes.

té en 1990)", $A F D I$, vol. 37, 1991, pp. 119-137. Sur le caractère nouveau de ce Comité, voir spécialement, pp. 120 et ss. et, pour une analyse plus récente, Stromseth, J. E., "An Imperial Securiy Council? Implementing Security Council Resolutions 1373 and 1390", American Society of International Law, Poceedings of the 97th Annual Meeting, 2003, pp. 41-45, et Tomuschat, C., Human Rights, Between Idealism and Realism, Oxford, Oxford University Press, 2003, p. 90.

13 Le Comité des sanctions en question a été créé par la résolution 1267 du Conseil de Sécurité en 1990. D'autres résolutions ont suivi, dans le but de préciser les pouvoirs et le fonctionnement du comité.

14 Fremuth, M. et Griebel, J., "On the Security Council as a Legislator: A Blessing or a Curse for the International Community?", Nordic Journal of International Law (NJIL), no. 76, 2007, p. 344. 


\section{LE PHÉNOMÈNE DES LISTES NOIRES \\ OU DE L'EFFET REDOUTABLE \\ DE LA COOPÉRATION INTERNATIONALE}

Avant même d'approfondir la question sur le plan strictement juridique, il faut procéder à un constat. Comme le rappelle E. Rosand, ${ }^{15}$ parmi les nombreux défauts de la liste noire, et parmi les raisons des réticences des Etats à transmettre des noms, l'accent a été mis sur le discrédit que l'affirmation d'un lien avec Ben Laden porte sur la personne et sur sa famille. ${ }^{16}$ Il s'agit, en effet, d'une source supplémentaire de stigmatisation ${ }^{17}$ de certaines populations s'ajoutant à celles qui, enracinées dans le passé colonial, nourrissent déjà suffisamment l'imaginaire collectif.

\section{La procédure d'inscription sur les listes noires du Comité des Sanctions de l'ONU: l'exemple de Monsieur Yusuf}

Ces noms sont fournis par les Etats. Leur insertion dans la liste n'est accompagnée d'aucune étude approfondie sur la pertinence du lien établi

15 Rosand, E., op. cit., note 8, p. 751. Dans le même sens, Hinijosa Martínez, L. M., "Las sanciones del Consejo de Seguridad contra Al Qaeda y los talibanes: ¿son realmente inteligentes?", Revista Española de Derecho Internacional (REDI), 2006, vol. LVIII, no. 2 , p. 762, d'après lequel le déficit en termes de crédibilité dont souffre le Comité peut induire les Etats à "hacer la vista gorda", autrement dit à fermer les yeux; ce qui, évidemment, compromet l'efficacité du système.

16 Certains auteurs, par exemple Nalin, E., "Misure europee anti-terrorismo e diritti umani”, La Comunità internazionale, 2004, vol. LIX, no. 2, p. 303, parlent à ce propos d'un véritable dommage à la vie de relation.

17 Voir en ce sens Gonzáles, M. "El idealismo estadounidense y la generación de la imagen del enemigo", en Z. Zeraoui et F. T. Montiel T. (eds.), La guerra contra el terror. Estados Unidos, Afganistán y la lucha contra el terrorismo, México, Instituto Tecnológico de Monterrey, Ariete, 2006, p. 91, où on souligne comment, aux U.S.A., l'attitude des médias a généré une "xenofobia contra 'los árabes' y la religión musulmana". Pour le contexte britannique, et notamment sur les effets de la nouvelle politique en matière de (retrait de la) nationalité, voir Walker, C., "The Treatement of Foreign Terror Suspects", The Modern Law Review, vol. 70, no. 3, 2007, pp. 427-457, où, p. 439, l'auteur souligne que les nouvelles mesures "will inevitably discriminate against persons of minority racial or ethnic origins who have settled in the United Kingdom and have assumed dual nationality". A ce propos on peut parler d'un "syndrome" des listes noires... Pour une étude approfondie du système américain du blacklisting, voir Peed, M. J., "Blacklisting as Foreign Policy: the Politics and Law of Listing Terror States", Duke Law Review, 2005, vol. 54, pp. 1296-1354. 
entre l'individu ou l'entité et les réseaux terroristes. ${ }^{18}$ Aux personnes incluses dans la liste s'appliquent des sanctions économiques, notamment le gel des fonds. De telles sanctions ne sont pas à strictement parler des sanctions pénaux, ce qui, d'après $\mathrm{P}$. Weckel, ${ }^{19}$ semble exclure l'exigence $\mathrm{du}$ contradictoire. Pourtant, tout en admettant une telle qualification, elles ont des "effets similaires à une condamnation pénale". ${ }^{20}$ Indépendamment de leur nature, ces sanctions sont appliquées par les Etats. Or, il est important de reconstruire le processus qui mène de la proposition des noms par les Etats à l'exécution des sanctions. Ce processus peut être reconstruit à partir de l'affaire Yusuf.

Le nom de Monsieur Yusuf a été proposé, avec celui d'autres ressortissant suédois, par les Etats-Unis. ${ }^{21}$ Le Comité des Sanctions de l'ONU

18 Rosand, E., op. cit., note 8, p. 748: "in practice, submission of names... often contained minimal personal information and did not generally include explanation of the connection the individual or entity and Osama bin Laden, or members of Al Qaeda and the Taliban". De fait, comme le rappelle Koskenniemi, M., op. cit., note 12, p. 137, "le comité a été tributaire de renseignement fournis par les participants à l'action d'une manière qui ne rehausse pas sa crédibilité". Andersson, T. et al., "EU Blacklisting: the Renaissance of Imperial Power, but on a Global Scale", European Business Law Review, 2003, vol. 14, no. 2, p. 111, insistent sur le caractère simplement conjectural des connexions, souvent très indirectes, que l'on reconstruit entre l'individu inscrit sur la liste et le réseau terroriste.

19 Weckel, P., "Note sous TPICE, 21 septembre 2005", RGDIP, 2005, vol. 3, p. 964.

20 Voeffray, F. "Le Conseil de sécurité de l'ONU: gouvernement mondial, législateur ou juge? Quelques réflexions sur les dangers de dérives", en M. G. Kohen (ed.), Promoting Justice, Human Rights and Conflict Resolution through International Law/La promotion de la justice, des droits de l'homme et du règlement des conflits par le droit international: liber amicorum Lucius Caflisch, Leiden, Nijhoff, 2007, p. 1205. En ce sens voir aussi Hinijosa Martínez, L. M., op. cit., note 15, p. 761: "el paso del tiempo puede convertir una medida cautelar en una sanción penal".

21 Rosana, E., op. cit., note 8, p. 749, rappelle que, dans les semaines qui ont suivi le 11 septembre, tout le monde s'est empressé à faire confiance à la liste de noms proposée par le Etats-Unis, qui était la même de l'Executive Order no. 13. 224 des USA (66 Fed. Reg. 49,077 du 25 Septembre 2001). Sur le fait que ce sont surtout les USA qui transmettent les noms, voir aussi Voeffray, F., op. cit., note 20, p. 1206. Pour une reconstruction précise des éléments de fait ayant justifié (ou censés justifier) l'inscription sur la liste de M. Yusuf, nous renvoyons à Miller, E. "The Use of Targeted Sanctions in the Fight Against International Terrorism - What About Human Rights?", American Society of International Law, Poceedings of the 97th Annual Meeting, 2003, pp. 48 et ss. 
l'a donc, automatiquement, ${ }^{22}$ inclus dans sa liste. ${ }^{23}$ En effet l'inscription sur la liste est presque "tacite": "si dans un délai de 48 heures, aucun membre du Conseil de Sécurité ne s'est opposé à l'inscription, celle-ci devient effective. Une fois un nom inscrit sur la liste, il n'est possible de le retirer qu'avec l'accord de tous les membres du Conseil de Sécurité". ${ }^{24}$ L'Union européenne s'est chargée de centraliser la mise en œuvre, à l'échelle européenne, des résolutions du Conseil de Sécurité. Cela s'est traduit par l'adoption, entre autres, d'un règlement communautaire, ${ }^{25}$ n'ayant d'autre objet que celui de reproduire ladite liste. Les Etats membres de l'Union européenne, face à l'adoption de ce règlement communautaire, se sont vus "dans l'obligation" d'y donner pleine exécution. Ils ont ainsi procédé au gel des fonds de Monsieur Yusuf. Celui-ci estimant injustifiée l'inscription de son nom sur ladite liste, et donc injustifiée la sanction, a formé un recours en annulation devant le juge communautaire. Formellement l'acte juridique appliqué par les Etats membres, l'acte fondant la validité de la sanction, est le règlement communautaire. Cependant, compte tenu du fait que les institutions communautaires, notamment le conseil, n'ont exercé aucun pouvoir d'appréciation, le juge communautaire a estimé qu'il n'avait aucune compétence pour juger de la pertinence de l'inclusion du nom de Monsieur Yusuf dans la liste.

\section{L'incompétence du tribunal et l'effet de licéité: l'interprétation des articles 103 de la Charte et 307 du TCE}

Le tribunal de première instance a insisté sur le devoir de loyauté de l'Union européenne à l'égard des engagements que les Etats membres ont assumé en signant la Charte des Nations Unies. C'est en effet celle-ci

22 Voeffray, F., op. cit., note 20, p. 1204. C'est-à-dire: “sans se donner les moyens nécessaires pour vérifier de manière fiable la responsabilité des personnes visées".

23 Le 19 octobre 2001, le Comité des sanctions a publié un nouvel addendum à sa liste incluant, entre autres, le nom de Monsieur Yusuf.

24 Voeffray, F., op. cit., note 20, p. 1206.

25 Il s'agit du règlement (CE) n 2062/2001 de la Commission, du 19 octobre 2001, modifiant, pour la troisième fois, le règlement n 467/2001 (JO L 277, p. 25). C'est l'annexe I de ce règlement qui contient la liste des personnes, des entités et des organismes visés par le gel des fonds. Aux termes de l'article 10, paragraphe 1, du même règlement, la Commission est habilitée à modifier ou à compléter ladite annexe I sur la base des décisions du Conseil de sécurité ou du Comité des sanctions. 
qui donne pouvoir au Conseil de Sécurité pour prendre toutes les mesures nécessaires au maintien de la paix et de la sécurité internationaux.

Le tribunal épouse la thèse défendue par les institutions dont le point de vue est ainsi résumé: ${ }^{26}$

le Conseil et la Commission soulignent que, en leur qualité de membres des Nations unies, les Etats membres de la Communauté ont accepté d'appliquer sans réserve les décisions prises en leur nom par le Conseil de sécurité, dans l'intérêt supérieur du maintien de la paix et de la sécurité internationales (voir article 24, paragraphe 1, et article 25 de la Charte des Nations unies). Les obligations qui incombent à un membre de l'ONU en vertu du chapitre VII de la Charte des Nations unies prévaudraient sur toutes les autres obligations internationales auxquelles il pourrait être soumis. L'article 103 de la Charte permettrait ainsi d'écarter toute autre disposition du droit international conventionnel ou coutumier en vue d'appliquer les résolutions du Conseil de sécurité, créant par là même un "effet de licéité”.

Ce point de l'arrêt mérite que l'on s'y arrête car l'existence d'une telle primauté ne va pas de soi. Il faut d'abord distinguer deux formes de primauté: d'une part, on peut estimer que le droit de la Charte prime sur le droit de communautaire; d'autre part, on peut aller plus loin et affirmer que toute norme juridique exprimée par une résolution du Conseil de Sécurité s'impose à la communauté et prime logiquement sur le droit communautaire, primaire et dérivé.

Afin de préciser ces aspects il faut revenir rapidement à la disposition du traité de laquelle on tire la primauté. La question en effet, n'est pas tant celle de la primauté du droit de l'ONU qui se fonde sur l'article 103 de la Charte et qui s'adresse aux Etats. Le vrai problème est celui de la soumission du droit communautaire, non pas à la Charte mais aux résolutions du Conseil de Sécurité. Or, indépendamment du contenu des résolutions qui nous intéressent une telle soumission est difficilement défendable.

26 Point 156 de l'arrêt Yusuf, cité. Sur l'interprétation de l'article 103, et sur ce que la primauté ainsi instituée pourrait légitimer en matière de protection des droits de l'homme, voir Paolo, P., "Azioni di forze istituite o autorizzate delle Nazioni Unite davanti alla Corte europea dei diritti dell'uomo: i casi Behrami e Seramati", RDI, vol. 90, afl. 3, 2007, pp. 681-704, spécialement p. 696, et infra, où il s'agira d'analyser les hiérarchies normatives qui semblent découler de la décision du Tribunal. 
Précisons que la soumission de la communauté à la Charte, en tant que telle, peut également poser un problème, ${ }^{27}$ mais la question est sans doute plus complexe. Il nous semble plus utile d'insister sur une thèse plus humble, et contester la soumission de la Communauté au droit issu des résolutions du Conseil de sécurité. ${ }^{28}$

Il est utile de prendre comme point de départ l'une des rares études affirmant que l'analyse du tribunal va "dans la bonne direction". ${ }^{29}$ D'après certains commentateurs de l'arrêt Yusuf, il serait en effet "impensable de voir les Etats membres de l'Union européenne échapper à leurs obligations en vertu de la Charte des Nations Unies par le biais de leur participation à un organisme international régional". ${ }^{30}$ Cette affirmation repose sur un présupposé: que les résolutions en question expriment "des obligations découlant de la Charte". ${ }^{31}$ La question semble alors être celle du sens à attribuer au mot "découler", ou plus précisément celle de la qualification du chemin permettant de passer de la charte aux résolutions du Conseil de Sécurité. Le choix du verbe découler semble privilégier une thèse forte: le droit issu des résolutions de l'ONU serait en quelque sorte implicite dans les principes de la charte, il en découlerait comme une conséquence logique. Dans ce cas, en effet, la primauté de la charte s'étendrait automatiquement à l'ensemble des normes exprimées par les résolutions. Or, il est toutefois légitime de douter que les résolutions, dont le contenu peut être le plus varié et qui, surtout, sont adoptées par une minorité d'Etats dans des situations parfois caractérisées par l'ur-

27 Labayle, H., "Architecte ou spectatrice? La Cour de Justice de l'Union dans un espace de liberté, sécurité et justice”, Revue trimestrielle de droit européen (RTDE), 2006, vol. 42, p. 40, constate ironiquement: "dans une vision irénique de la succession de la Communauté aux Etats membres, le Tribunal consacre donc la primauté d'un droit dont elle n'est ni actrice ni destinataire".

28 A supposer en effet que l'on adopte une vision moniste avec primauté du droit international, comme le souligne Roldán Barbero, J., "La interacción entre las normas internas e internacionales en el acervo jurídico europeo", Revista de Derecho Comunitario Europeo, vol. 6, no. 13, 2002, p. 701, "la opción monista no es observada en relación a los actos unilaterales adoptados por otras organizaciones internacionales"; le fait que l'Union européenne ne soit pas membre de l'ONU constitue, d'après l'auteur, une difficultés supplémentaire.

29 Stangos, P. et Gryllos, G., "Le droit communautaire à l'épreuve des réalités du droit international: leçons tirées de la jurisprudence récente relevant de la lutte contre le terrorisme international", Cahiers de droit européen, no. 3-4, 2006, p. 466.

30 Idem.

31 Ibidem, p. 465. 
gence, puissent "découler" de la charte. Pour utiliser un vocabulaire kelsénien, la validité des résolutions est dynamiquement et non statiquement fondée sur le droit de la charte..$^{32}$ Comme le rappelle dans une étude récente Robert Kolb, ${ }^{33}$ le Conseil de Sécurité agit en vertu d'une habilitation, exprimée par les articles 41 et 42 . Dans l'hypothèse, analysée par l'auteur, d'un conflit entre une résolution et, par exemple, l'interdiction de génocide, le conflit se donnerait entre la résolution et le jus cogens et non entre la charte (sur laquelle pourtant se fonde la résolution) et le jus cogens: cette dernière solution, en effet, "ne rendrait pas compte du grand degré de latitude que le chapitre VII laisse à la décision du conseil". ${ }^{34}$ Le lien entre les résolutions du Conseil de Sécurité et la charte est donc purement dynamique. Si les normes exprimées par les résolutions "découlaient" de la charte, alors il faudrait affirmer, dans l'hypothèse évoquée par Robert Kolb, que de la charte découle, entre autres, une norme incompatible avec l'interdiction du génocide. Cela, en dépit du fait qu'on puisse s'évertuer à en démontrer la plausibilité, semble pour le moins contre intuitif.

Il nous semble donc possible de douter de l'automaticité de la primauté des normes issues des résolutions. Or, tout en admettant une telle primauté, il ne faut pas oublier qu'elle s'appliquerait logiquement aux Etats membres, mais non à la communauté. Il est alors nécessaire de franchir un autre pas, commandé par l'article 307 du traité instituant les communautés européennes. D'après le tribunal:

[s]'agissant plus particulièrement des rapports entre les obligations des Etats membres de la communauté en vertu de la charte des Nations unies et leurs obligations en vertu du droit communautaire, il convient d'ajouter que, aux termes du premier alinéa de l'article $307 \mathrm{CE}$, "[1]es droits et obligations résultant de conventions conclues antérieurement au 10. janvier 1958 ou, pour les États adhérents, antérieurement à la date de leur adhésion, entre un ou plusieurs États membres, d'une part, et un ou plusieurs

32 Sur cet aspect de l'interprétation kelsénienne des pouvoirs conférés par la Charte au Conseil de sécurité, voir Garibian, S., Le crime contre l'humanité au regard des principes fondateurs de l'Etat moderne. Naissance et consécration d'un concept, Thèse, Droit, Université de Paris X - Nanterre et Université de Genève, 2007, p. 428.

33 Kolb, R., "Conflits entre normes de 'jus cogens"”, in Droit du pouvoir, pouvoir du droit: mélanges offerts à Jean Salmon, Bruxelles, Bruylant, 2007, p. 499.

34 Ibidem, p. 499. 
États tiers, d'autre part, ne sont pas affectés par les dispositions du présent traité".

...cette disposition a pour objet de préciser, conformément aux principes du droit international, que l'application du traité CE n'affecte pas l'engagement de l'État membre concerné de respecter les droits des États tiers résultant d'une convention antérieure et d'observer ses obligations correspondantes. ${ }^{35}$

Encore une fois la question est celle de savoir quelles sont, exactement, les obligations correspondant aux engagements des Etats membres.

Il faut procéder à une première distinction: d'une part, il existe des normes connues, c'est-à-dire exprimées par des dispositions de conventions internationales publiées avant l'entrée en vigueur du traité; dans ce cas, la question ne se pose pas: de telles obligations conventionnelles, pour ainsi dire, ont été acceptées par la communauté. Cela étant dit, les Etats concernés, d'après le deuxième alinéa de l'article 307, “[d]ans la mesure où ces conventions ne sont pas compatibles avec le présent traité... recourent à tous les moyens appropriés pour éliminer les incompatibilités constatées". Ce qui signifie que cet article ne proclame pas une primauté de toute obligation conventionnelle antérieurement souscrite; elle se limite à accepter un état de fait, précisant que les Etats mettront tout en œuvre pour mettre fin à l'incompatibilité, par exemple en renégociant un traité ou en le dénonçant. Il faut également évoquer l'hypothèse d'un traité qui violerait des normes impératives: dans ce cas, en vertu de la Convention de Vienne, les obligations conventionnelles en question cesseraient d'obliger les Etats. Or, il est inutile d'aller plus loin dans cette voie, car les normes dont il s'agit dans l'affaire Yusuf ne sont pas des normes conventionnelles.

En effet, outre de telles normes issues de dispositions conventionnelles "connues", il faut envisager l'existence de normes qui seraient le produit d'organes "normolateurs" créés par des conventions et donc "inconnues" au moment de l'adhésion à la communauté. C'est de toute évidence le cas des normes exprimées par les résolutions du Conseil de Sécurité.

A ce état il faut procéder à une autre distinction, ayant trait à l'objet des conventions: on doit distinguer les conventions qui règlent les rap- 
ports juridiques entre Etats, des conventions qui instituent des organisations internationales. En ce sens l'ONU et la Communauté doivent être traitées de la même manière. Si le droit communautaire a été, dès le début, conçu comme un ordre dynamique, dans lequel les normes de droit dérivé s'imposent directement en vertu du traité, on ne peut dire la même chose pour le système juridique onusien.

On ne peut déduire, de l'éventuelle primauté de la charte, la primauté de toutes les normes exprimées par des résolutions du Conseil de Sécurité, sachant que cet organe n'est pas conçu comme normolateur et, surtout, qu'à supposer qu'il soit ainsi, il n'existe aucun moyen de contrôler la conformité de ces normes aux principes de la charte. ${ }^{36}$

Autrement dit: si les Etats membres de 1'Union européenne sont soumis au droit dérivé, c'est parce qu'on suppose que le droit dérivé soit conforme aux principes exprimés par les traités (et aux principes généraux du droit communautaire dont font partie les principes issus de la Convention européenne des droits de l'homme). Si ce n'est pas le cas, il suffit de former un recours en annulation. Si ce recours n'est pas formé ou si le juge communautaire constate la légalité de l'acte, les Etats doivent se soumettre. Mais, à supposer que les résolutions expriment des normes, qu'en est-il de la possibilité pour les Etats, y étant soumis, de solliciter un contrôle de légalité?

Beaucoup d'arguments qui, à notre avis, font douter du fait que l'article 307 puisse être interprété comme donnant priorité à n'importe quelle résolution de l'ONU. Mais admettons qu'une telle priorité soit envisageable. Compte tenu du pouvoir discrétionnaire du Conseil de Sécurité, il serait impossible de savoir quel sera le droit applicable. Cela signifierait que l'article 307 permettrait d'introduire des normes juridiques dont le contenu pourrait être le plus varié, à la seule condition qu'elle soient exprimées par des résolutions du Conseil de Sécurité agissant en vertu du chapitre VII de la charte. Il est alors important d'insister sur l'atteinte inévitable au principe de sécurité juridique: attribuer au Conseil de sécurité un pouvoir créateur de droit et octroyer aux normes ainsi produites un statut privilégié, les rendant obligatoires pour tous, reviendrait à introduire parmi les sources suprêmes du droit -international, communautaire et national - un élément absolument arbitraire ou au moins imprévisible. 
Indépendamment d'une telle remarque, une interprétation de l'article 307 tenant compte du contexte de son élaboration permet de relativiser la primauté des dites résolutions. C'est le tribunal lui-même qui montre le chemin, lorsque, pour appuyer la thèse de la primauté, évoque l'article 297, inséré, d'après les juges,"afin de respecter la règle de primauté". ${ }^{37}$ En vertu de cet article, les Etats membres "se consultent en vue de prendre en commun les dispositions nécessaires pour éviter que le fonctionnement du marché commun ne soit affecté par les mesures qu'un Etat membre peut être appelé à prendre... pour faire face aux engagements contractés par lui en vue du maintien de la paix et de la sécurité internationale".

On comprend aisément l'utilité d'un tel article dans le raisonnement du tribunal: la référence à la paix et à la sécurité internationale semble rendre solide la thèse de la primauté. Cependant, on ne saurait passer sous silence le fait que cet article a pour objet le marché commun. Autrement dit, il n'est pas question d'atteintes aux droits fondamentaux, mais simplement d'affectation du fonctionnement du marché commun. Or, si on se situe au moment où les articles cités par le tribunal ont été rédigés, force est de constater que les engagements auxquels se réfèrent les articles 307 et 297, ne sont évoqués que dans la mesure où ils peuvent affecter la communauté économique, les relations commerciales.

Autrement dit, alors même qu'on admettrait le caractère constitutionnel de l'article 307, on devrait poser la question suivante: cet article est-il hiérarchiquement supérieur à tous les autres, et notamment à ceux qui proclament le respect des droits fondamentaux?

Une interprétation discutable mais raisonnable aurait dû, au moins, mettre en balance les intérêts en conflit. Ce serait toutefois taire que l'article 307 vise le maintien des relations commerciales avec les pays tiers car, il ne faut pas l'oublier, l'objet du droit communautaire n'était qu'économique. Si cet article avait été rédigé en même temps que l'article 6eme. du TUE, on aurait pris soin d'y introduire une clause de sauvegarde. Or, probablement, le TPICE, en invoquant le jus cogens, tente d'introduire ce type de clause. C'est comme si le tribunal disait: l'article 307 attribue une primauté au droit de l'ONU, mais il faut l'interpréter à la lumière de la Convention de Vienne sur le droit des traités, étant précisé qu'un traité n'est pas obligatoire s'il contrevient au jus cogens. Nous re- 
viendrons sur cet aspect original de l'arrêt; il nous suffira de constater, pour l'instant, que l'effet de ce tortueux raisonnement est une sorte d'immunité juridictionnelle dont bénéficient les résolutions du Conseil de Sécurité.

A partir du constat d'un tel "effet de licéité", le seul recours possible semble alors un recours devant le Conseil de sécurité lui-même.

\section{L'intervention passive de l'Union européenne dans l'exécution des résolutions onusiennes, ou comment priver les individus (et les Etats) de tout recours effectif}

La situation est pernicieuse dans la mesure où l'Union européenne intervient à un état intermédiaire, à savoir entre les résolutions onusiennes et l'exécution étatique des sanctions. En effet, si l'Union européenne n'était pas intervenue, la situation aurait été bien différente. Indépendamment des différences qui existent entre les Etats au sujet de leur option constitutionnelle, moniste ou dualiste, et, surtout, de la prétendue primauté des résolutions du Conseil de Sécurité, il nous semble pertinent de penser qu'en cas d'acte international soulevant des doutes quant au respect des droits fondamentaux, les juridictions constitutionnelles des Etats européens n'hésiteraient pas à le signaler et à s'opposer à l'adoption des instruments de réception. ${ }^{38}$ C'est pour cette raison que certains auteurs ont par ailleurs insisté sur l'effet bénéfique d'une application nationale des sanctions en question. ${ }^{39}$

38 Sur ce point voir Brown, R., "Kadi v. Council of the European Union and the Commission of the European Communities: Executive Power and Judicial Supervision at European Level", European Human Rights Law Review, vol. 4, 2006, pp. 456-469. D'après l'auteur, si l'on peut admettre que, "from an international law standpoint, the UN Charter has primacy over domestic rules", cela n'exclut pas "the possibility that a Member State may assert the primacy of its own sovereignty over a resolution of the Security Council" (p. 462); plus précisément: "a domestic court may assert that Security Council resolutions have no effect in internal law" (p. 466). Dans le même sens Vandepoorter, A., "L'application communautaire des décisions du conseil de sécurité", AFDI, 2006/1, p. 128: “rien ne s'oppose à ce qu'un juge, quel qu'il soit, puisse contrôler une résolution, une fois admis qu'il s'agit bien d'un acte juridique. Et, à l'évidence; c'est le cas".

39 Andersson, T. et al., op. cit., note 18, p. 121: "It would also allow them to have a proper system of national judicial control of such measures. Furthemore, UN sanctions would be made subject to domestic constitutional requirement and the gouvernement would have to assume the political responsability for implementation". Un tel point de vue sem- 
Autrement dit: l'article 103 de la charte, peut-il réellement être interprété comme obligeant les Etats à tirer un trait sur toutes les obligations qu'ils ont contractées à partir d'instrument internationaux eux mêmes fondés sur la Charte ou qui découlent directement de leurs constitutions incluant souvent des déclarations de droits fondamentaux? ${ }^{40}$

Si l'on peut répondre par la négative, force est de constater que l'intervention purement "formelle" de l'Union européenne, se limitant à recopier les listes des noms préparées par le Comité des Sanctions a pour effet de mettre les Etats devant un acte communautaire qui, en vertu du principe de primauté, s'impose directement. Un Etat qui n'appliquerait pas les sanctions économiques à Monsieur Yusuf violerait le droit communautaire et serait donc susceptible de faire l'objet d'une procédure en manquement d'Etat. Alors qu'un Etat qui ne se conformerait pas à une résolution de l'ONU (phénomène, de fait, relativement fréquent) n'aurait à craindre que des rétorsions politiques. ${ }^{41}$ Certains auteurs affirment sans ambages que les Etats "peuvent toujours refuser d'appliquer les résolutions du Conseil de Sécurité qu'ils jugent illégales". ${ }^{42}$ En effet, d'un point de vue plus général, il ne faut pas négliger le principe cardinal du droit international, à savoir celui du consentement des Etats. Ceux-ci ont certes consenti à la charte, mais ont-ils consenti à ce que le Conseil de

ble confirmé par une décision récente d'une juridiction turque, libre de toute soumission au droit communautaire. Le Conseil d'Etat turc, en effet, dans une décision du 4 juillet 2006, a annulé la décision de gel de fond prise par l'Etat turque, à l'encontre d'une personne listée par le Comité des sanctions, pour manque de preuves, du fait que personne n'a pu mettre à sa disposition les éléments permettant d'établir le lien avec le reseau Al-Quaida (DOC. S/2006/750, 20 septembre 2006, anexe III, p. 54, cité par Hinijosa Martínez, L. M., op. cit., note 15, p. 753).

40 En ce sens, voir Miller, E., op. cit., note 21, p. 50: "it would seem more than legalistic to assume that Article 103 can absolve the organisation or its member states of their human rights obligations".

41 Sur ce point voir Brown, R., op. cit., note 38, p. 462: si l'on peut admettre que, "from an international law standpoint, the UN Charter has primacy over domestic rules", cela n'exclut pas "the possibility that a Member State may assert the primacy of its own sovereignty over a resolution of the Security Council"; plus précisément: "a domestic court may assert that Security Council resolutions have no effect in internal law" (p. 466).

42 Bore Eveno, V., "Le contrôle juridictionnel des résolutions du Conseil de sécurité: vers un constitutionnalisme international?”, RGDIP, vol. 4, 2006, p. 858. 
sécurité se comporte en législateur universel? ${ }^{43}$ Alors même que la réponse est, ou devrait être, négative, il serait cependant myope de ne pas voir que la réalité est toute autre: un tel pouvoir est exercé ${ }^{44}$ et contribue à l'émergence de ce que certains auteurs ont pu appeler une véritable hégémonie juridique. ${ }^{45}$

La situation qui découle de cette configuration juridique est on ne peut plus paradoxale: l'Union européenne ne joue aucun rôle, dans l'affaire Yusuf, dans l'appréciation du lien existant entre une personne et le réseau Al-Quaida, et pourtant son intervention passive, voir transparente, rend par là même incontestables les appréciations (parfois absentes) du Comité des sanctions. En cas de contestation, la volonté d'un seul Etat, par exemple les Etats-Unis, en vertu du droit de veto dont disposent les membres permanents du Conseil de Sécurité, suffira à archiver la question. ${ }^{46}$

Or, il est acquis en doctrine que la Cour internationale de justice, juge institué par la charte, nie sa compétence pour contrôler la légalité des résolutions du Conseil de Sécurité. ${ }^{47}$ Le seul recours possible est

43 Sur ce point, voir l'analyse très convaincante de M. Fremuth - J. Griebel, "On the Security Council as a Legislator: A Blessing or a Curse for the International Community?", NJIL, 76, 2007, p. 354: “Apart from the fact that the SC accordingly does not enjoy a competence to legislate, such a power would also be highly problematic with respect to the principle of consent in international law. Deriving from the principle of sovereign equality enshrined in Article 2(1) of the UN-Charter, a State is only bound by rules it has consented to".

44 Fremuth, M. et Griebel, J., op. cit., note 43, p. 355: "there are many fields of law where breaches can give rise to threats to international peace and security, the SC would enjoy a wide competence in lawmaking and could feel inclined to enact more and more legal acts".

45 Idem: "The emergence and development of public international law would the mostly depend on the five permanent members of the SC that possess a veto-power, risking the development of a legal hegemony".

46 La question qui se pose est alors celle de savoir quelles sont les limites à l'exercice du droit de veto. En ce sens voir L. Paulus, A., "Jus Cogens in a Time of Egemony and Fragmentation. An attempt at a Re-appraisal", NJIL, vol. 74, 2005, p. 325: "it remains unclear wheter superpower status or permanent membership in the Security Council may confer a veto regarding the emergence of new jus cogens, too".

47 Sur la question du contrôle de la légalité des résolutions du Conseil de sécurité, voir Bedjaoui, M., "Du contrôle de légalité des actes du Conseil de sécurité", in Nouveaux itinéraires en droit. Hommage à François Rigaux, Bruylant, Bruxelles, 1993, p. 69; id., "Un contrôle de la légalité des actes du Conseil de sécurité est-il possible?", in $\mathrm{Le}$ chapitre VII de la Charte des Nations Unies, Paris, Pédone, 1995, p. 258; Pellet, A., 
alors une sorte de recours gracieux devant le Comité des sanctions. En effet celui-ci est chargé de mettre à jour les listes de noms et, en accomplissant ce travail, il est à même de rayer des noms. Il semblerait alors exister un moyen d'action. Cependant les personnes dont le nom est sur la liste n'ont aucun accès direct au comité. Ils ne peuvent que solliciter leur Etat (de résidence ou d'appartenance) afin que celui-ci, à son tour, sollicite un réexamen du cas individuel. ${ }^{48}$

Or, ces perplexités ont donné lieu à une véritable réflexion, au sein même de l'ONU ${ }^{49}$ et à une jurisprudence plus nuancée de la part du juge communautaire..$^{50}$

"Peut-on et doit-on contrôler les actions du Conseil de sécurité?", in Le chapitre VII de la Charte des Nations Unies, ouv. cit., p. 224. Ce dernier auteur semble implicitement admettre (p. 227) la possibilité d'un contrôle da la validité des résolutions du Conseil de sécurité par n'importe quel juge ayant à appliquer le droit international: "Les résolutions du Conseil de sécurité font partie du droit international et les obligations en découlant ont, dans la sphère du droit international lui-même, une valeur supérieure en vertu des dispositions de l'article 103 de la Charte. Encore faut-il que ces obligations découlent de résolutions juridiquement valides. Les tribunaux nationaux peuvent s'en assurer et en ont même le devoir, faute de quoi ce n'est pas 'le droit international' qu'ils mettraient en œuvre”. Voir enfin les développements des juges d'appel du Tribunal pénal international pour l'ex-Yugoslavie dans l'affaire Tadic, d'après lesquels "ni la lettre ni l'esprit de la Charte ne conçoivent le Conseil de sécurité comme legibus solutus (échappant à la loi)" (arrêt Dusko Tadic, 2 octobre 1995, IT-94-1, paragraphe 28). Pour une analyse approfondie du contrôle que, sur ce fondement, les juges pénaux semblent avoir exercé sur l'action du Conseil de sécurité, voir Garibian, S., op. cit., note 32, pp. 424 et ss.

48 Comme le constate Rosand, E., op. cit., note 8, p. 750: "without legal recourse to challenge their inclusion on the UN list or the asset freeze, the Somali Swedes were sensed to have been convicted of and punished for a crime without due process". Dans le même sens Miller, E., op. cit., note 21, p. 49; l'auteur met en exergue, p. 48, le contexte kafkaïen d'un éventuel recours: comment prouver l'innocence des personnes inscrites lorsqu'on ignore les raisons justifiant leur inscription? Comme le rappelle Florent, J. L., op. cit., note 3, p. 114, une telle réalité est d'autant plus kafkaïenne qu'il n'est pas rare d'être sur les listes pour une simple question d'homonymie.

49 Weckel, P., op. cit., note 19, p. 965, évoque le travail de l'équipe de surveillance du Comité des sanctions aboutissant à des conclusions prônant l'automaticité de la transmission de la demande de radiation (l'auteur cite à ce propos la lettre du président du Comité au Conseil de sécurité du 15 février 2005, S/2005/83, spécialement l'annexe II, par. 56). Sur la prise en compte par le Secrétaire général de l'Onu des exigences visant une amélioration des procédés, et la mise en place des garanties minimales voir Voeffray, F., op. cit., note 21, p. 1208.

50 Pour une étude synthétique de l'ensemble de cette jurisprudence voir P. Aust, H., "Between Self-Assertion and Deference: European Courts and their Assessment of UN 


\section{L'émergence d'un droit subjectif à l'exercice d'une forme sui generis de protection diplomatique}

Le Tribunal de Première Instance des Communautés Européennes a eu l'occasion de revenir sur la question des sanctions économiques individuelles. Une première fois dans un contexte presque identique à celui de l'affaire Yusuf; une deuxième fois, dans des affaires où il était question de contrôler la légalité de sanctions prononcées sur le fondement d'un acte communautaire traduisant l'exercice, par les institutions communautaires, d'un pouvoir d'appréciation. Les juges de Luxembourg ont ainsi pu, dans l'arrêt Ayadi du 12 juillet 2006, ${ }^{51}$ reconnaître l'insuffisance des voies de recours auprès de l'ONU et envisager, par conséquent, un droit subjectif de toute personne figurant sur les listes à exiger de son Etat (de résidence ou d'appartenance, cela reste peu clair) d'agir auprès du Comité des Sanctions afin d'obtenir la radiation. ${ }^{52}$ D'autre part, les mêmes juges, ont, dans l'arrêt $O M P I$ du 12 décembre 2006,,$^{53}$ annulé l'acte communautaire dans la partie concernant les requérants, entre autre au motif de l'absence de motivation suffisante concernant le lien entre

Security Council Resolutions", Anuario Mexicano de Derecho Internacional, vol. VIII, 2008, pp. 64 et ss. (affaire Ayadi) 67 et ss. (affaire OMPI).

51 Arrêt Ayadi, op. cit., note 5.

52 Le Tribunal communautaire semble avoir trouvé une inspiration dans une décision belge rendue par le Tribunal de première instance de Bruxelles, le 18 février 2005, dans l'affaire Nabil Sayadi et Patricia Vinck c. Etat Belge. Le Tribunal dégage trois points essentiels, qui vont dans le même sens que la jurisprudence communautaire: "il ne peut être contesté que le Tribunal est sans juridiction pour censurer ou s'immiscer dans les décisions d'une organisation internationale... Il appartient au dit Comité des sanctions, et à lui seul, d'inscrire ou non... Il ne peut pas non plus être fait grief aux autorités... belges d'avoir tiré les conséquences de cette décision". A partir d'un tel triple constat, les juges condamnent cependant l'Etat belge à demander au Comité la radiation de la liste. Il est utile de préciser qu'une telle demande de l'Etat belge est restée sans suite. Sur cette décision, voir Arcari, M., "Sviluppi in tema di tutela dei diritti di individui iscritti nelle liste di comitati delle sanzioni del Consiglio di sicurezza", RDI, 2007, afl. 3, pp. 674 et ss.

53 TPICE, aff. T-228/02, Organisation des Modjahedines du peuple d'Iran / Conseil de l'Union européenne. 
les entités visées et les réseaux terroristes. Dans le même esprit d'une telle dernière motivation, l'Avocat général de la Cour de justice des Communautés européennes, saisie d'un pourvoi dirigé contre l'arrêt Kadi (affaire jointe à celle de Monsieur Yusuf), a développé, dans ses conclusions, ${ }^{54}$ un argumentaire allant dans le sens de l'annulation du règlement communautaire reproduisant la liste dressée par le Comité des sanctions. En l'état actuel du droit et de la jurisprudence, la seule nouveauté est constituée par l'existence d'un droit subjectif à l'exercice d'une protection de la part de l'Etat. Nouveauté qui a suscité quelques perplexités et un peu d'enthousiasme. ${ }^{55}$

\author{
III. BOSPHORUS, YUSUF, BERHAMI: OU DE L'EFFET \\ "BLANCHISSANT" DU CHAPITRE VII DE LA CHARTE DE L'ONU \\ ET DE LA COOPÉRATION INTERNATIONALE
}

La formule blanchissante magique semble être l'appel aux exigences de la coopération internationale; c'est en effet en s'appuyant sur les "devoirs" découlant d'une telle coopération que les juges de Strasbourg et de Luxembourg établissent et justifient le régime de ce qui semble être une véritable immunité juridictionnelle dont bénéficient les résolutions du Conseil de Sécurité.

54 Conclusions de l'Avocat général Poiares Maduro, présentées le 16 janvier 2008, dans l'affaire C402/05.

55 Sur la nature d'un tel recours, à mi-chemin entre un recours gracieux et une nouvelle forme de protection diplomatique, voir Symon, D. et Mariatte, F., "Le 'droit' à la protection diplomatique: droit fondamental en droit communautaire?", Europe, 2006, p. 7; les auteurs soulignent la nouveauté de la décision du Tribunal de première instance des communautés européennes, tout en en montrant les limites du point de vue du droit international en vigueur. D'autres auteurs n'ont pas hésité à envisager une obligation pour la Communauté elle-même d'assurer une telle protection (Stangos, P. et Gryllos, G., "Le droit communautaire à l'épreuve des réalités du droit international: leçons tirées de la jurisprudence récente relevant de la lutte contre le terrorisme international", Cahiers de droit européen, vol. 3-4, 2006, p. 472 et ss.). 
La plupart des commentateurs ont déjà mis en exergue et approfondi les aspects les plus importants des jurisprudences Bosphorus ${ }^{56}$ et Yusuf..$^{57}$ Nous nous limiterons alors à insister sur cette sorte de nouveau système juridique international qui en découle et sur l'évolution jurisprudentielle, en particulier l'affaire Berhami jugée par la Cour Européenne des Droits de $1^{\prime} H o m m e,{ }^{58}$ qui indirectement le confirme.

\section{Le nouvel ordre juridique international: le juge communautaire inventeur de nouvelles hiérarchies normatives}

Le premier constat est celui de l'existence d'une hiérarchie. ${ }^{59}$ Plus exactement il faudrait parler de l'existence de deux hiérarchies. L'une

56 Benoît-Rohmer, F., “A propos de l'arrêt Bosphorus Air Lines: l'adhésion contrainte de l'Union à la Convention", $R T D H$, vol. 64, 2005, pp. 827-853; Ciampi, A., op . cit., note 11; Cannizzaro, E., op. cit., note 11, p. 762; Jacqué, J.-P., "Droit communautaire et convention européenne des droits de l'homme: l'arrêt Bosphorus, une jurisprudence Solange II de la Cour européenne des droits de l'homme", RTDE, vol. 41, afl. 3, 2005, pp. 756-767; Hoffmeister,F., "Bosphorus Hava Yollary Turizm et al. v. Ireland. ECHR Review of National Measure Enforcing EC Regulation Implementing UN Security Council Decision", AJIL, 2006, vol. 100, afl. 2, pp. 442-449.

57 Jacqué, J.-P., "Le Tribunal de première instance face aux résolutions du Conseil de sécurité des Nations unies: 'Merci monsieur le Professeur'", L'Europe des libertés, no. 19, 2006, voir www.leuropedeslibertes.u-strasbg.fr. Symon, D. et Mariatte, F., op. cit., note 55, pp. 4-7. Conforti, B. "Decisioni del consiglio di sicurezza e diritti fondamentali in una bizzarra sentenza del Tribunale comunitario di primo grado", Il diritto dell'Unione europea, 2, 2006, p. 333; A. Atteritano, "Il congelamento dei beni quale strumento di lotta al terrorismo: il mantenimento della pace e della sicurezza internazionale e il rispetto dei diritti dell'uomo nell'ottica del Tribunale di I grado CE, tra jus cogens, risoluzioni vincolanti del Consiglio di Sicurezza e principi comunitari fondamentali", Giurisprudenza costituzionale, vol. 2, 2006, pp. 1699-1731; Brown, R., op. cit., note 38.

$58 \mathrm{CEDH}$, Grande chambre, citée. Pour un commentaire approfondi de la décision, Palchetti, P., "Azioni di forze istituite o autorizzate delle Nazioni Unite davanti alla Corte europea dei diritti dell'uomo: i casi Behrami e Seramati", RDI, vol. 90, afl. 3, 2007, pp. 681-704. Pour un résumé de la décision en langue française, Gabard, V., "Incompétence rationae personae de la Cour pour les actions directement imputables à l'ONU", voir www.sfdi.org/actualites/a2007/Sentinelle\%20113.htm\#cedh.

59 Comme le rappelle Jacqué, J.-P., "Droit constitutionnel, Droit communautaire, CEDH, Charte des Nations Unies. L'instabilité des rapports de système entre ordres juridiques", $R F D C$, vol. 69, janvier 2007, p. 31: "La hiérarchie des normes est au cœur de la solution retenue par le Tribunal". Dans le même sens, Weckel, P., op. cit., note 19, p. 957 , où il opère le constat suivant: "une juridiction internationale spécialisée de plus, et non une des moindres, affirme sans ambiguïtés la hiérarchisation du droit international". 
concernant les actes normatifs, l'autre concernant les normes juridiques. L'action du Conseil de Sécurité est d'autant plus libre qu'elle fait l'objet d'un contrôle, ne serait-ce qu'incident, par une juridiction qui, en dépit d'une compétence qui semble avoir suscité quelques perplexités auprès de la doctrine, de fait érige une hiérarchie de normes au sommet desquelles figurent les normes dites de jus cogens. ${ }^{60}$

Il s'agit bien de deux hiérarchies. ${ }^{61}$ La première fait primer les actes du Conseil de Sécurité sur les actes des institutions communautaires. Il s'agit de la hiérarchisation des pouvoirs respectifs de deux organisations internationales. Les pouvoirs du Conseil de Sécurité sont en effet qualifiés d" "impératifs" par la CEDH dans son arrêt Berhami. Un tel caractère impératif semble alors exclure toute possibilité de "contrôle juridictionnel". Mais, afin de limiter le caractère abdicataire de l'attestation d'une telle hiérarchie, les juges communautaires inventent une autre hiérarchie tout aussi fantaisiste, ${ }^{62}$ mettant le jus cogens au-dessus de tout et notamment au-dessus des résolutions du Conseil de Sécurité. ${ }^{63}$

60 Sur la notion de jus cogens la littérature est immense. On se limitera à renvoyer à un travail récent qui a le mérite, à partir de l'analyse de la jurisprudence la plus récente, de montrer la nature insaisissable et les fonctions variables du jus cogens: Focarelli, C., "I limiti dello jus cogens nella giurisprudenza più recente", Rivista di diritto internazionale, 2007, vol. 3, pp. 637-656. Pour une étude approfondie du concept voir Kolb, R., Théorie du Ius Cogens international. Essai de relecture du concept, Paris, PUF, 2001; Rivier, R., Droit impératif et juridiction internationale, Thèse, Droit, Paris 2, 2001. Voir aussi, pour un travail moins récent mais tout aussi intéressant, Gómez Robledo, A., El Ius Cogens internacional (Estudio histórico-crítico), México, Universidad Nacional Autónoma de México, 1982. Pour une étude approfondie du lien entre ce concept et celui de hiérarchie des normes en droit international, Shelton, D., "Normative Hierarchy in International Law", AJIL, vol. 100, afl. 2, 2006, pp. 291-323.

61 Bien que dédiée à l'étude du droit constitutionnel, l'étude de L. Depussay, "De la hiérarchie des normes à la hiérarchie des pouvoirs", Droit prospectif. Revue de la Recherche Juridique, vol. 3, 2007, pp. 1177-1193, est très éclairante, surtout dans là mesure où l'auteur constate que "l'agencement des pouvoirs se désolidarise de la théorie traditionnelle de la hiérarchie des normes" (p. 1186).

62 D'après Labayle, H., op. cit., note 27, p. 38: "la hiérarchie dessinée à ce propos laisse... l'observateur dubitatif". Jacqué, J.-P., "Droit constitutionnel, Droit communautaire", op. cit., note 59, p. 33, parle alors de "primauté sous réserve". Si une telle primauté du droit de l'ONU semble privilegier la thèse d'une vision moniste professée par le Tribunal, d'autres points de l'arrêt semblent désavouer une telle hypothèse. En ce sens, Atteritano, A., op. cit., note 57, p. 1714.

63 D'après Weckel, P., op. cit., note 19, p. 965, "le jus cogens est vraisembleblement indispensable à la coexistence du droit international et du droit communautaire, parce 
Il est important de préciser que le tribunal, après avoir affirmé ce devoir de respecter le jus cogens, insiste sur le caractère improbable d'une violation: "le droit international permet ainsi de considérer qu'il existe une limite au principe de l'effet obligatoire des résolutions du Conseil de Sécurité: elles doivent respecter les dispositions péremptoires fondamentales du jus cogens. Dans le cas contraire, aussi improbable soit-il, elles ne lieraient pas les États membres de l'ONU ni, dès lors, la Communauté". ${ }^{64}$ Il s'agit d'une attitude relativement classique, déjà exprimée par le juge ad hoc Lauterpacht:65 "il n'y a pas lieu d'envisager que le Conseil de Sécurité se trouve jamais adopter délibérement une résolution qui ferait ouvertement et directement fi d'une règle du jus cogens ou qui prescrirait une violation des droits de l'homme. Mais on ne saurait exclure la possibilité que, par inadvertance ou de façon imprévisible, le Conseil de sécurité adopte une résolution qui aboutirait à une telle situation". Or, souvent lorsque l'on pose la question de la légalité des résolutions du Conseil de sécurité, c'est plus pour rapidement la confirmer ${ }^{66}$ que pour mener à bien une analyse juridique sérieuse; autrement dit, il s'agirait de ce que l'on appelle une question rhetorique.

Un commentateur ${ }^{67}$ a pu remarquer que la définition de ce qui est protégé par le jus cogens, en matière de propriété, ou de "substance incompressible" de ce droit, est élaborée, bien que de manière implicite, en tenant compte des intérêts concurrents, notamment la lutte contre le terrorisme. ${ }^{68} \mathrm{Il}$ semble en découler, indépendamment du caractère critiquable des arguments du tribunal, qu'il n'y a pas nécessairement de contra-

qu'il permet à ce dernier de plier devant la primauté du droit international sans trahir l'essence de la Communauté européenne en tant qu'état de droit".

64 Arrêt Yusuf, op. cit., note 5, paragraphe 281.

65 Opinion individuelle, CIJ, Application de la convention pour la prévention et la répression du crime de génocide, Bosnie-Herzgovine c. Serbie-et-Monténégro, ordonnance du 13 septembre 1993 (nouvelles demandes en indication de mesures conservatoires), I.C.J Reports, 1993, pp. 440-441, paragraphe 102 (nous soulignons).

66 Comme le souligne Verhoeven, J., Les nullités en droit des gens, Cours et travaux de l'IEHI, Paris, Pedone, 1981, p. 13, lorsque la Cour internationale de justice semble controler les résolutions du Conseil de sécurité, elle s'attache surtout à "asseoir dans la mesure du possible la validité des décisions de l'Organisation des Nations Unies, fût-ce par prétérition".

67 Tancredi, A., 'L'emersione dei diritti fondamentali 'assoluti' nella giurisprudenza comunitaria", $R D I$, vol. 3, afl. 3, 2000, pp. 644-692.

68 Ibidem, p. 691. 
diction entre le fait d'affirmer le caractère absolu de certains droits et le fait d'avoir recours à la balance pour en définir la portée. Il s'agit de deux étapes du même iter argumentatif. ${ }^{69}$ Il s'agirait alors de ce que l'on a pu appeler une hiérarchie axiologique mobile ${ }^{70}$ — qui s'ajouterait (ou conduirait), à celle, peu défendable, d'ordre formel— ${ }^{71}$ dans laquelle le principe "préféré" ne l'est que dans le cas concret, à partir d'une opération d'arbitrage entre les valeurs en conflit. Comme le soulignait déjà le doyen Vedel, "le juge opère une pesée... entre les valeurs en présence. Il concèdera plus ou moins à l'une ou à l'autre des normes en conflit en prenant des critères très variés". ${ }^{72}$ Mais l'arbitrage n'est pas explicite: il reste masqué par les opérations de qualification juridique, notamment de l'atteinte aux droits fondamentaux dont il est question. ${ }^{73}$ Autrement dit: le "périmètre" de l'inviolabilité peut difficilement être mesuré a priori. ${ }^{74}$

Aussi vraisemblable qu'elle soit dans un univers purement normatif, où le Sollen ne croiserait jamais le Sein, le contrôle par le TPICE de la légalité des résolutions du Conseil de Sécurité prend l'allure d'une mise en scène aussi séduisante que vaine si les concepts prétendent à être signifiants, force est de constater que dans l'histoire du droit, l'existence

69 Ibidem, p. 692.

70 Sur la notion de hiérarchie axiologique mobile, voir Champeil-Desplat, V., "Raisonnement juridique et pluralité des valeurs: les conflits axio-téléologiques de normes", Analisi et Diritto, 2001, p. 66; Puppo, A., "Sur les conflits entre principes constitutionnels: le raisonnement des juges entre rhétorique des valeurs morales et logique des propositions juridiques", à paraître. Voir aussi Drago, G., "La conciliation entre principes constitutionnels", Dalloz, 1991, Chron., pp. 265 et ss.; Turpin, D., "Le traitement des antinomies des droits de l'homme par le Conseil constitutionnel", Droits, 2, 1985, pp. 85 et ss.; Rousseau, D., "Droit du contentieux constitutionnel”, 6eme. éd., Paris, Montchrestien, pp. 126 et ss.

71 En effet, d'après Tancredi, A., op. cit., note 67, p. 678, les intérêts protégés par le jus cogens sont "protetti da norme collocate al vertice di gerarchie formali o assiologiche".

72 Vedel, G., "La place de la Déclaration de 1789 dans le bloc de constitutionnalité", in La déclaration des droits de l'homme et du citoyen et la jurisprudence, Paris, PUF, 1989 , p. 59.

73 Tancredi, A., op. cit., note 67, p. 689: "L'attività di qualificazione può, dunque, 'mascherare' un'operazione di 'secondo livello', un'operazione di 'arbitraggio'”.

74 Ibidem, p. 683. Comme le souligne L. Paulus, A., op. cit., note 46, p. 329, à propos de la difficulté d'identifier les normes ayant la qualité de jus cogens: "The problem, however, lies in the interpretation of facts pointing to their violation. In some cases, in particular in the cas of agression after the beginning of the 'global war on terror', the situation has become muddier rather than clearer". 
d'un contrôle, et donc d'une hiérarchie entre normes, ${ }^{75}$ a été rarement affirmée indépendamment de la possibilité de son exercice "utile". ${ }^{76}$

Il est inutile d'insister sur ce point car personne en doctrine ne semble avoir pris au sérieux la tentative du TPICE de "contrôler" le Conseil de Sécurité. La doctrine a déjà eu le loisir de stigmatiser les erreurs juridiques, ${ }^{77}$ notamment à propos de la Convention de Vienne, à laquelle tous les Etats membres ne sont pas partie, ${ }^{78}$ et à propos des normes de jus cogens (que le tribunal de première instance semble trouver là où personne, avant lui, n'avait cru en voir $)^{79}$ sur lesquelles il serait alors aussi irrespectueux que redondant de revenir.

Il faut se limiter à l'essentiel. Pourquoi le juge communautaire aurait-il, après avoir nié son pouvoir pour le faire, exercé ne serait-ce que "pour la forme" un tel contrôle? La seule explication rationnelle semble découler directement de la jurisprudence Bosphorus sur la protection équivalente. Pour ne pas démentir la confiance que le juge de Strasbourg semble accorder au juge communautaire, il fallait montrer que, tout comme dans l'affaire jugée en juin 2005 par le CEDH, dans l'affaire Yusuf, les impératifs de la coopération internationale devaient s'inscrire dans le respect de ce que l'on a appelé l'ordre public européen. ${ }^{80}$ Cela revient à

75 Sauf à distinguer, comme le fait Bore Eveno, V., op. cit., note 42, p. 837, entre "hiérarchie" et "hiérarchie effective".

76 Sur le caractère purement irréaliste d'un tel contrôle, dont l'effectivité porterait par ailleurs atteinte au multilatéralisme sur lequel se fonde la coopération internationale et notamment l'action de l'ONU, voir Jacqué, J.-P., op. cit., note 59, pp. 32 et 33.

77 Voir en particulier Jacqué, J.-P., op. cit., note 57, pp. 6 et ss.; mais surtout Labayle, H., op. cit., note 27, qui parle, p. 37, “d'une jurisprudence discutable, frappée d'insuffisance manifeste dans la maîtrise des concepts fondamentaux du droit international et peu convaincante pour le maintien à niveau des droits fondamentaux".

78 Voir J. Glennon, M.,"De l'absurdité du droit impératif (jus cogens)", RGDIP, vol. 3, 2006, pp. 529 et ss. et, en particulier, p. 533, sur l'interprétation "légère" du Tribunal (arrêt Yusuf, paragraphe 278), qui ne s'interroge pas sur l'applicabilité de la Convention de Vienne, non rétroactive, à la Charte des Nations unies, qui la précède.

79 Weckel, P., op. cit., note 19, p. 963, salue en ces termes la vision futuriste du Tribunal: "Les jugements du 21 septembre 2005 annoncent donc le commencement d'une nouvelle ère dans l'application du jus cogens qui n'est plus celle de la jurisprudence $\mathrm{Fu}$ rundzija. Le jus cogens enfle dans des proportions impressionnantes et à une vitesse exponentielle".

80 Jacqué, J.-P., op. cit., note 59, p. 33, souligne que "le souci du Tribunal est de montrer qu'il existe des limites infranchissables en matière de protection des droits fondamentaux". 
dire que, alors même que l'action du Conseil de Sécurité ne saurait être entravée, elle doit s'inscrire dans les pouvoirs (impératifs) que lui attribue la charte et respecter le jus cogens.

\section{Le contrôle de l'action du Conseil de Sécurité: des chemins} tortueux entre impératifs moraux et raisons d'Etats

Il faut alors distinguer deux formes de contrôle qui semblent émerger de la jurisprudence Yusuf et de la jurisprudence Berhami. Si le tribunal, dans l'arrêt Yusuf, affirme sans ambages le devoir de respecter le jus cogens, la CEDH, elle, semble en revanche laisser la porte ouverte à un contrôle qui s'effectuerait, non pas à la lumière du jus cogens, mais à la lumière de la charte elle-même. Si le conseil détient des pouvoirs impératifs, c'est parce qu'il agit pour préserver la paix. D'après la Cour, "il convient d'accorder encore plus de poids au caractère impératif du but premier de l'ONU et, par voie de conséquence, des pouvoirs accordés au Conseil de sécurité en vertu du chapitre VII pour atteindre ce but". ${ }^{81}$ Les juges déduisent, ensuite, du caractère impératif que "la Convention ne saurait s'interpréter de manière à faire relever du contrôle de la Cour les actions et omissions des Parties contractantes couvertes par des résolutions du Conseil de Sécurité et commises avant ou pendant de telles missions"; ce qui revient à affirmer que les actes des Etats, à supposer qu'ils aient pu violer des droits protégés par la Convention "sont primordiaux pour l'accomplissement effectif par le Conseil de sécurité du mandat qui lui est conféré par le chapitre VII, et donc pour la réalisation par l'ONU du but impératif de maintien de la paix et de la sécurité qui lui est assigné". 82

Selon une telle reconstruction, si on démontrait que l'action du conseil ne s'inscrit pas dans les buts et principes de la charte, alors elle perdrait toute légitimité: "Si le chapitre VII constitue le fondement de la délégation des pouvoirs de sécurité du Conseil de sécurité, pareille délégation doit être suffisamment limitée pour rester compatible avec le degré de centralisation de la sécurité collective assurée par le Conseil de Sécurité qui est constitutionnellement nécessaire en vertu de la charte et, plus particulièrement, pour que les actes de l'entité déléguée soient attri-

81 CEDH, Berhami, op. cit., note 4, paragraphe 148.

82 Ibidem, paragraphe 149. 
buables à l'ONU". ${ }^{83}$ Il s'agirait alors de distinguer entre pouvoir discrétionnaire et pouvoir arbitraire du Conseil de sécurité et donc de "vérifier qu'il n'est pas allé au-delà de ce pouvoir qui lui a été attribué". ${ }^{84} \mathrm{Si}$ les actes ne sont pas attribuables à l'ONU, on pourrait alors parler d'une sorte de voie de fait internationale et, dans ce cas, l'immunité juridictionnelle tomberait.

L'existence de deux hiérarchies ne fait que reposer une question classique ayant animé la théorie du droit d'orientation positiviste: quelle est la nature du droit international? Est-ce vraiment du "droit"? Or la hiérarchie qui se fonde sur les pouvoirs impératifs du Conseil de sécurité s'explique aisément si l'on conçoit l'ordre international comme étant un réseau de pouvoirs, avec une dimension purement politique. ${ }^{85} \mathrm{Si}$ en revanche on admet que l'ordre international est un "droit", alors il faut imaginer que même le Conseil de Sécurité est soumis au respect de certaines normes supérieures, non seulement celles de la charte mais aussi celles, aussi obscurs que soient leur fondement et leur contenu, de jus cogens.

En dernière analyse, si, comme cela est difficilement contestable, le jus cogens, en tant que droit impératif, semble se rapprocher dangereusement d'un corpus de normes morales ${ }^{86}$ s'imposant aussi bien aux Etats

83 Ibidem, paragraphe 132. D'après Palchetti, P., op. cit., note 58, p. 691, la Cour semble se réserver le droit de vérifier la compatibilité de l'autorisation en vertu de laquelle les Etats agissent au nom de l'ONU avec le système des Nations unies. Autrement dit: l'auteur relève "che la Corte sembra riconoscere una propria competenza a giudicare la legittimità delle misure adottate dal Consiglio di sicurezza". Cela étant dit, constate l'auteur, aucune indication n'est donnée pour savoir à partir de quels critères une telle appréciation pourrait être effectuée.

84 Vandepoorter, A., op. cit., note 38, p. 131.

85 Dans ce cas, comme le précise Bore Eveno, V., op. cit., note 42, p. 829, "lorsque les actes du Conseil de sécurité sont contestés, il est difficile de ne pas y voir la manifestation d'un désaccord politique, plus que le souci de voir assuré le respect d'un principe de légalité".

86 Voir en ce sens, l'affirmation, fort évocatrice, du Tribunal pénal international pour l'ex-Yougoslavie à propos de l'humanisation du droit international: "Cette tendance inscrit dans les normes juridiques le concept 'd'impératif catégorique', formulé par Kant dans le domaine de la morale... la plupart des normes du droit international humanitaire, notamment celles qui prohibent les crimes de guerre, les crimes contre l'humanité et le génocide, sont des normes impératives du droit international ou jus cogens, c'est-à-dire qu'elles sont impérieuses et qu'on ne saurait y déroger" (Jugement TPIY Ch. II, Mirjan Kupreskic et al., 14 janvier 2000, IT-95-16, paragraphes 518 et ss.). D'après Garibian, S., 
qu'aux individus et aux organisations internationales, alors le constat est assez paradoxal. La prolifération des juridictions internationales censées "appliquer des normes juridiques" semble montrer que le juridique occupe une place bien mince. ${ }^{87}$ La notion d'ordre public semble témoigner d'une telle ambiguïté, d'un tel constant glissement entre la raison d'Etat (ou la raison des Etats membres permanents du Conseil de sécurité) et les droits de 1'homme. ${ }^{88}$ Les expressions "ordre public européen", 89 "ordre public international", ${ }^{90}$ semblent confirmer une telle confusion sémanti-

op. cit., note 32, p. 521: "Il n'en reste pas moins que la référence expresse au jus cogens... traduit le choix des juges de "positiver" les principes moraux invoqués en 1945: le droit applicable est fondé sur des normes dont la valeur impérative ne serait pas issue de principes naturels, mais de la volonté supposée des Etats souverains membres de la communauté internationale". Il s'agit, en dernière analyse, comme le résume avec acuité Dupuy, P.-M., "La communauté internationale. Une fiction?", in Droit du pouvoir, pouvoir du droit: mélanges offerts à Jean Salmon, Bruxelles, Bruylant, 2007, p. 386, de deux options théoriques: celle de la fiction (la volonté supposé de la Communauté internationale) et celle de l'impératif (moral) kamtien. Sur les difficulté, à supposer que l'on adopte un point de vue positivste, soulevées par l'invocation du jus cogens, voir. Glennon, M. J., op. cit., note 78, p. 531. L'auteur, après avoir rappelé quelques évidences, à savoir que "le système juridique international n'applique pas de règles morales aux Etats" et que "les principes moraux ne sont pertinents que dans la mesure où on leur donne 'une expression suffisante sous forme juridique", conclut qu'une telle "grundnorm positiviste représente une vérité encombrante pour les tenants du jus cogens": "la réalité est que le jus cogensne peut être reconcilié avec les fondements d'un ordre juridique international positiviste" (p. 532).

87 L'effet de l'invocation du jus cogens comme source d'obligatoriété pourrait avoir comme effet paradoxale de déclasser les autres normes juridiques, non "impératives". En ce sens voir L. Paulus, A., op. cit., note 46, p. 322: "The introduction of a 'higher' category of international law may well be used to 'downgrade' ordinary rules, to the point when only jus cogens rules appear to be binding on States, both internationally and domestically".

88 En ce sens voir Cannizzaro, E., "A Machiavellian Moment? The UN Security Council and the Rule of Law", International Organisation Law Review, vol. 2, 2006, p. 200, note 24. Comme le rappelle. Dupuy, P.-M., op. cit., note 86, p. 375, en reprenant les mots de Charles Peguy, "tout commene par la mystique et tout fini par de la politique".

$89 \mathrm{CEDH}$, Bosphorus, paragraphe 156: d'après les juges, la "Convention en tant qu'instrument constitutionnel de l'ordre public européen dans le domaine des droits de l'homme l'emporterait sur l'intérêt de la coopération internationale". Le conditionnel étant de rigueur vu que la condition, en l'espèce, serait celle de l'insuffisance manifeste de protection des droits fondamentaux. La notion est rappelée dans l'arrêt Berhami, paragraphe 145 , où on reprend textuellement l'extrait ci-dessus.

90 Le TPICE, dans l'affaire Yusuf, introduit la notion d'ordre public international pour affirmer (paragraphe 277) que "le Tribunal est néanmoins habilité à contrôler, de manière 
que. Dans le langage juridique "ordinaire", on associe la notion d'ordre public à celle de police, la deuxième étant notamment "missionnée" pour préserver le premier. En ce sens le Conseil de Sécurité est un acteur de premier plan, en tant que gendarme ou pompier international. ${ }^{91}$ Mais dans les arrêts de la CEDH l'ordre public se définit en tant que "protection des droits fondamentaux". Or, dans cette configuration, c'est l'ordre public (au sens traditionnel) qui justifie des atteintes à l'ordre public (au sens de la CEDH). ${ }^{92} \mathrm{Il}$ n'est pas étonnant que chaque branche du droit ${ }^{93}$ redéfinisse à sa guise cette notion; la passerelle, vraisemblablement, est fournie par la notion de dignité, ${ }^{94}$ à la fois "minimum incompressible", ${ }^{95}$

incidente, la légalité des résolutions en cause du Conseil de sécurité au regard du jus cogens, entendu comme un ordre public international qui s'impose à tous les sujets du droit international, y compris les instances de l'ONU, et auquel il est impossible de déroger".

91 Certains auteurs ont pu insister sur ce point, en soulignant la dérive qui tend à faire du Conseil de sécurité une sorte de législateur universel, voir de juge universel: Voeffray, F., op. cit., note 20, surtout p. 1200, où l'auteur estime que le Conseil de sécurité devrait se limiter à son rôle institutionnel de "gendarme chargé du maintien de l'ordre, ou... pompier oeuvrant à la prévention et à l'extinction des incendies".

92 Comme le rappelle le Gouvernement britannique (CEDH, Berhami, paragraphe 115): "La requête soulève des questions fondamentales concernant la relation entre la Convention (traité régional et 'instrument constitutionnel de l'ordre public européen') et le système universel de maintien de la paix internationale, dans lequel le Conseil de l'Europe joue un rôle important. Par principe, il ne conviendrait pas de superposer cette structure régionale en matière de droits de l'homme à une force de maintien de la paix établie par une organisation universelle. Cela irait à l'encontre de l'ordre public auquel la Cour se réfère fréquemment, et risquerait en outre de causer de graves difficultés aux Etats contractants lorsqu'ils participent à des opérations de maintien de la paix de l'ONU ou à d'autres opérations multinationales en dehors de leurs territoires" (nous soulignons).

93 A titre d'exemple, au sein même du droit de l'Union européenne, la CJCE a eu à préciser que cette notion n'a pas le même sens selon que l'on se situe dans le droit communautaire "classique" ou dans le droit "communautarisé" de Schengen. La solution de la Cour a privilégié l'interprétation la plus favorable aux libertés fondamentales, et obligé par conséquent les Etats à interpréter restrictivement les pouvoirs de police qu'ils tirent des Accords de Schengen (CJCE, Grande chambre, 31 janvier 2006, Commission c./ Espagne; aff. C 503/03).

94 La doctrine a brillamment analysé le concept de dignité. Voir Favoreu, L., "Note sous Conseil Constitutionnel n. 94-343/344 DC du 27 juillet 1994", RFDC, vol. 20, 1994, pp. 799-811; Mathieu, B., "Note sous Conseil Constitutionnel n. 94-359 DC du 19 janvier 1995", Les petites affiches, 7 juin 1995, n. 68; id., "La dignité de la personne humaine: quel droit? quel titulaire?", Dalloz, 1996, pp. 282-286.

95 L'expression est de Rivero, J., "L'entreprise et les libertés publiques", Droit social, 1982, vol. 4, p. 423. 
absolu et transcendant les autres droits, ${ }^{96}$ et "composante de l'ordre public". ${ }^{97}$ Une telle imbrication devient patente en droit pénal international où la notion d'ordre public international est invoquée, à la fois, comme justifiant la mise en place d'un "ordre répressif international" et comme visant la protection des droits de la personne humaine. ${ }^{98}$ Or, si l'on parle depuis longtemps de normes juridiques en réseau, d'internormativité, ici on va encore plus loin. Le droit, s'inscrivant dans la coopération internationale, devient purement circulaire, et dans ce cercle qui a bien peu de vertu, les juridictions européennes ne peuvent que s'exhiber en faisant d'improbables pirouettes. Or, le problème, au-delà du respect ponctuel des droits individuels, devient alors celui de la crédibilité même de l'Europe en tant que "vitrine" des droits de l'homme. ${ }^{99}$ Comment continuer à promouvoir les droits de l'homme alors qu'au cœur même de l'Europe les juridictions les plus engagées dans la protection des droits

96 Mathieu, B., "La dignité de la personne humaine: quel droit? quel titulaire?”, op. cit., note 94, p. 285.

97 Conseil d'Etat, Assemblée, 27 octobre 1995, Cne de Morsang-sur-Orge et Ville d'Aix-en-Provence: Rec., p. 372 (affaire dite du "lancer de nains"). Pour une analyse de la notion de dignité, de ses rapports avec l'ordre public, et du caractère discutable de la décision du Conseil d'Etat, voir Lebreton, G., "Ordre public et dignité de la personne humaine: un problème de frontière", in M.-J. Redor (ed.), L'ordre public: Ordre public ou ordres publics? Ordre public et droits fondamentaux, Bruxelles, Bruylant, 2001, pp. 353-367; Levinet, M., "Dignité contre dignité. L'épilogue de l'affaire du 'lancer de nains' devant le Comité des Droits de l'Homme des Nations Unies", Revue trimestrielle des droits de l'Homme, 2003, pp. 1017-1042. Pour une étude de droit comparé, qui insiste sur l'absurdité d'une dignité objective qui serait subjectivement déterminée par les juges, et sur la confusion opérée par le Conseil d'etat français entre ordre public et ordre moral, voir Resta, G., "La disponibilità dei diritti fondamentali e i limiti della dignità (note a margine della carta dei diritti)", Rivista di Diritto Civile, 2002, p. 801, spéc, pour le cas de la France, pp. 837 et ss.

98 Sur cette interférence entre répression et protection et, plus précisément sur le lien étroit entre la notion de dignité, de jus cogens et l'émergence d'un ordre public international, voir Garibian, S., op. cit., note 32, spécialement pp. 207 et ss., sur le lien indissoluble entre répression internationale, sauvegarde des droits de l'homme et maintien de la paix internationale, pp. 234 et ss., à propos de la jurisprudence pénale française et de l'émergence de l'idée d'un ordre répressif international, et pp. 621 et ss., sur le lien entre dignité, ordre public international et jus cogens (nous renvoyons à la note 374, p. 621, pour d'importantes références doctrinales).

99 En effet comme le souligne Shelton, D., op. cit., note 60, p. 311, la conclusion à laquelle aboutit le juge communautaire "call into question the entire framework of human rights guarantees established in Europe since the end of World War II". 
fondamentaux n'hésitent pas à se plier devant les exigences de la coopération internationale?

\section{La soumission de la protection des droits de l'homme aux exigences} du maintient de la paix et de la sécurité internationaux

Deux vérités semblent alors incontestables. D'abord, l'action du Conseil de Sécurité semble ne rencontrer aucun obstacle et, au contraire, avoir trouvé dans les institutions européennes un vassal, ${ }^{100}$ un exécutant (ou un "porte parole" voire un "prête-nom") dont la bonne réputation en matière de respect de droits fondamentaux semble à même de réaliser au mieux les objectifs propres au chapitre VII de la Charte de l'ONU, à savoir la sauvegarde de la paix et de la sécurité internationales et notamment la lutte contre le terrorisme.

Certains auteurs pourtant critiques à l'égard de la décision du Tribunal dans l'affaire Yusuf semblent plutôt enclins à admettre une suprématie du maintien de la paix: on accepte par conséquent que, si les circonstances l'exigent, le Conseil de sécurité puisse suspendre l'application de certains droits fondamentaux. ${ }^{101}$ Le vrai problème serait alors celui des limites du pouvoir discrétionnaire permettant une telle suspension car la primauté de "l'objectif Paix peut parfois s'opposer à l'objectif Droit et ouvrir la porte à un certain arbitraire". ${ }^{102}$ Or, à supposer que cela soit acceptable, du moins en principe, il faut néanmoins insister sur la nécessité qu'une telle suspension soit exceptionnelle et temporaire, ce qui n'est pas le cas en l'espèce. ${ }^{103}$ Autrement dit: il n'est pas acceptable de pous-

100 En ce sens, voir Eechkout, P., Does Europe's Constitution Stop at the Water's Edge?, 5th Walter van Gerven Lecture, Leuven Centre for a Common Law of Europe, 2005, p. 26: "Community order is a mere vassal of the UN Security Council. It is being commandeered by th UN and by the CFSP, and become an instrument for turning UN resolution into supreme law, a supreme law offering virtually no guarantees of judicial review, at any level".

101 Hinijosa Martínez, L. M., op. cit., note 15, p. 756: "no puede descartarse que, en el caso de que las circunstancias así lo exijan, el Consejo de Seguridad suspenda la aplicación de determinados derechos fundamentales".

102 Bore Eveno, V., op. cit., note 42, p. 845.

103 Hinijosa Martínez, L. M., op. cit., note 15, p. 761. 
ser ce type de raisonnement jusqu'à donner carte blanche pour violer les droits fondamentaux. ${ }^{104}$

En effet, alors même que l'on admettrait "le respect des droits fondamentaux demeure une obligation pour les Etats membres pour autant qu'il ne paraît pas susceptible de faire obstacle à la bonne exécutiton de leurs engagements en vertu de la Charte de Nations unies", ${ }^{105}$ et que donc les premiers doivent être pondérés ayant à l'esprit les impératifs découlant de la sécurité internationale, dans le cas d'une inscription dans la liste de nom du Comité "fondée sur des éléments faux ou inadéquats, la pondération entre l'objectif de la lutte contre le terrorisme international et les conséquences auxquelles les personnes inscrites sur ladite liste sont exposées est dénuée de toute pertinence". ${ }^{106}$ Autrement dit: "le gel de fonds d'une personne ne présentant aucun lien avec le terrorisme international n'est pas en rapport avec cette lutte"107 et ne peut donc, en aucun cas, être tolérée.

Une autre interprétation de la même configuration juridique serait celle, non pas de la suspension, mais plus simplement de la dérogation: le Tribunal nous enseignerait qu'il n'existe pas de droits individuels indérogeables; ce qui toutefois contredirait l'invocation par le même juge du jus cogens. ${ }^{108}$ Il n'y aurait donc ni suspension, encore moins violation: il s'agirait tout simplement de faire primer l'intérêt général que représente la sécurité internationale. ${ }^{109}$ Reste à expliquer l'invocation, par

104 Canor, I., “'Can Two Walk Together, Except They Be Agreed?' The Relationship Between International Law and European Law: The Incorporation of United Nations Sanctions Against Yugoslavia Into European Community Law Through the Perspective of the European Court of Justice", Common Market Law Review, vol. 35, afl. 1, 1998, p. 162 .

105 Stangos, P. et Gryllos, G., op. cit., note 29, p. 472.

106 Ibidem, p. 475.

107 Idem.

108 En ce sens voir l'analyse de Gallo Cobian, V., et al., "Las sanciones del Consejo de Seguridad de las Naciones Unidas y los derechos humanos. Relaciones peligrosas", Anuario Mexicano de Derecho Internacional, vol. VIII, 2008, p. 185.

109 D'après Atteritano, A., op. cit., note 57, p. 1719, c'est le Conseil de sécurité lui-même qui opère la pondération entre les droits fondamentaux et la paix internationale: "il fatto che la risoluzione in oggetto abbia avuto il consenso della Comunità internazionale, e che in sede di esecuzione in ambito UE nessuno degli Stati europei abbia svolto considerazioni critiche al riguardo, fa presumere che il c.d. balance compiuto dal $\mathrm{CdS}$ sia stato adeguato". Une telle affirmation affirme, comme le suggère Conforti, B., "Il ruolo del giudice nel diritto internazionale", European Journal of Legal Studies, vol. 1, 
le même juge qui affirme, du moins implicitement, le caractère dérogeable de tout droit fondamental, du jus cogens.

Une telle invocation pourrait être interprétée comme la simple manifestation d'un souhait: face au constat de la primauté des valeurs sécuritaires, le juge ne se résignerait pas au silence et, en mettant en scène un contrôle improbable des résolutions du Conseil de Sécurité envoie un message à la communauté internationale. Il est utile de rappeler, que l'on s'inscrit dans une tendance: "le jus cogens est progressivement utilisé dans des domaines où il n'est pas certains que l'on ait besoin de lui'. ${ }^{110}$ On n'as pas vraiment besoin de lui en tant que norme juridique, ${ }^{111}$ c'est pourquoi, "configuré comme principe de hiérarchie matérielle, le jus cogens en ressort dilué". ${ }^{112}$ Mais de fait il est, dans l'affaire Yusuf, invoqué "pour souligner l'importance d'une règle [et] n'apparaît plus alors que comme un argument d'autorité, flirtant avec la rhétorique". ${ }^{113}$ Or toute entreprise rhétorique suppose un auditoire. Dans ce cas, le destinataire du discours est la Cour Européenne des Droits de l'Homme; cela en raison de la bonne réputation dont jouissent les institutions européennes et notamment la cour de justice, telle que confirmée par la jurisprudence Bosphorus, dans laquelle les juges de Strasbourg constatent que le système communautaire assure une protection équivalente à celle assurée par la convention.

Dans l'affaire Bosphorus, il était question, comme dans l'affaire $Y u$ suf, de mesures, prises sur la base d'actes communautaires eux-mêmes donnant exécution à des résolutions du Conseil de Sécurité, portant atteinte au droit de propriété. Le juge communautaire ayant été saisi au préalable par le juge irlandais d'une question préjudicielle, la Cour de Strasbourg a estimé inutile de procéder à un examen du fond de la question. Dans le cas où une violation aurait été injustifiée ou non proportion-

no. 2, décembre 2007, p. 6, que "il problema dei rapporti tra prassi giurisprudenziale e prassi degli stati c'è e che esso potrebbe proficuamente costituire l'oggetto di uno studio approfondito di carattere scientifico".

110 Rivier, R., op. cit., note 60, p. 52.

111 En ce sens Shelton, D., op. cit., note 60, p. 305, "in reality... those norms most often identified as jus cogens are clearly accepted as customary international law and there are no persistent objectors"; d'où la question de l'auteur: qu'ajoute-t-on réellement en disant d'une norme qu'elle fait partie du jus cogens?

112 Rivier, R., op. cit., note 60, p. 53.

113 Idem. 
nelle aux buts (toujours ceux du chapitre VII de l'ONU) poursuivi, le juge communautaire aurait été en mesure de la constater et de tout mettre en œuvre pour y mettre fin.

Le système de voies de recours contentieuses proposé par le droit communautaire offre donc une protection équivalente. C'est le grand apport de la jurisprudence Bosphorus. Protection équivalente qui ne signifie autre chose qu'abdication des juges de Strasbourg. ${ }^{114}$ Certes, il serait injuste de taire que la cour prévoit des exceptions, un tel principe d'équivalence cessant d'opérer au cas où l'on constaterait une insuffisance manifeste de la protection des droits fondamentaux. ${ }^{115}$

Un auteur, en essayant de trouver un aspect encourageant dans la décision du tribunal communautaire, a pu, avec une pointe d'ironie, affirmer que "ce constat d'inapplicabilité de la Convention EDH, en parfaite méconnaissance de la jurisprudence de sa cour, a au moins un mérite: elle permettra effectivement de trancher la dernière question qu'avait éludée la Cour EDH dans sa jurisprudence Bosphorus". ${ }^{116}$

Ensuite, une telle coopération, dans le cadre de la lutte contre le terrorisme, semble reconnaître une sorte de jus ad bellum postmoderne; la guerre n'étant plus celle d'antan, une guerre entre Etats souverains, mais celle menée par une communauté internationale ${ }^{117}$ — dont le caractère

114 Le phénomène de l'abdication juridictionnelle semble tributaire, d'après certains auteurs, de la fragmentation du droit international et, spécialement, de la multiplication des juridictions internationales. Pour une réflexion générale sur la question, qui en minimise le caractère dramatique, voir Conforti, B., "Unité et fragmentation du droit international: 'Glissez, mortels, n'appuyez pas!'”, RGDIP, vol. 111, afl. 1, 2007, pp. 5-18. En effet, là où certains stigmatisent le risque de conflit d'interprétations, l'auteur met en évidence un "signe de vitalité du droit international" (p. 18). Pour une synthèse de la doctrine sur la question, nous nous permettons de renvoyer à la note 2, p. 5 , de ce remarquable travail. Pour l'analyse d'un exemple récent d'abdication, voir Humber, A., "L'attrait trompeur de la pluralité des juges internationaux: libres réflexions à partir de l'affaire du Liechtenstein devant le C.E.D.H. et la C.I.J.", Revue de droit international et de droit comparé, 84, 2007, 3, pp. 313-351.

115 Comme le précise Barreto, I. C., "La Cour européenne des droits de l'homme et le droit communautaire (quelques réflexions à propos de l'arrêt Bosphorus)", in M. G. Kohen (ed.), op. cit., note 20, p. 77: “il n'y a pas de certitudes permanentes parce qu'il n'y a pas de présomption irréfragables".

116 Labayle, H., op. cit., note 27, p. 40.

117 Mais, comme le souligne Voeffray, F., op. cit., note 20, p. 1202: "De quelle légitimité un organe à composition restreinte, où certains pays disposent d'un droit de veto, 
hybride et les formes juridiques changeantes lui permettent d'échapper à toute responsabilité, nationale ou internationale - ${ }^{118}$ contre des individus qui, sur la base de motivations souvent lacunaires, voire absentes, incarnent l'ancien rôle attribué aux pirates, hosti humani generis, ${ }^{119}$ en tant qu'ennemis déterritorialisés de la communauté internationale.

Une telle configuration ne peut alors que questionner sur les rapports classiques entre individu et Etat, entre Etat et communautés supra-étatiques (régionales ou internationales), et en dernière analyse sur le sens actuel de la protection internationale des droits de l'homme à laquelle la civilisation occidentale semble avoir donné tant d'importance et que, de fait, cette même civilisation semble vouloir mettre entre parenthèses. Il semblerait que la jurisprudence récente, y compris l'invocation rhétorique du jus cogens, ait contribué à faire tomber un masque dont se pare, avec orgueil, la communauté internationale. Comme l'affirme avec désenchantement, Raphalle Rivier, "le jus cogens remplit alors une fonction légitimante et intégratrice par la production de représentations unificatrices destinées à alimenter le mythe d'une société internationale dont aucun des principaux acteurs de la scène internationale ne rêve". ${ }^{120}$

Un regard vers le passé peut nous apporter quelques enseignements à propos de la tendance presque physiologique de l'Etat ou, aujourd'hui, de la communauté internationale à trouver une forme de cohésion dans la création d'un ennemi commun et une forme de légitimation dans l'offre de rédemption que l'on adresse à de tels ennemis.

\section{L'atteinte aux droits de l'étranger ennemi comme élément constitutif de l'Etat et de la communauté internationale}

Que l'on songe en premier lieu à la conquête de l'Amérique. Il suffira de rappeler le sens du requerimiento, sur lequel Todorov attire l'atten-

peut-il se prévaloir pour prétendre légiférer au nom de l'ensemble de la communauté internationale?".

118 En ce sens, voir Weil, P., "Cour général de droit international public", RCADI, vol. VI, tome 237, 1992, p. 311.

119 Voir sur la notion de hostis humani generis, Garibian, S., "Hostes humani generis. Les pirates vus par le droit”, Critique, 733-734, 2008, pp. 470-479.

120 Rivier, R., op. cit., note 60, p. 53. Un peu moins cinique est la réflexion, non moins lucide, de Dupuy, P.-M., op. cit., note 86, p. 376, à propos de la Communauté internationale, source du jus cogens: "une communauté dont chacun doute de la réalité mais ressent également le besoin". 
tion: une injonction adressée aux indiens à propos du fondement juridique de la conquête; il fallait informer les Indiens de ce fondement juridique. Or, on leur lisait le texte du requerimiento (au passage: on ne mentionne pas la présence d'interprètes...): "Si les Indiens se montrent convaincus à la suite de cette lecture, on n'a pas le droit de les prendre comme esclaves... Si cependant ils n'acceptent pas cette interprétation de leur propre histoire, ils seront sévèrement punis". ${ }^{121}$

La justification juridique ultime, élaborée notamment par Francisco de Vitoria, dégagée de toute consonance religieuse, se fonde sur le droit naturel. ${ }^{122}$ Les droits naturels de l'homme sont nés alors en tant que droits naturels des Etats souverains (parmi lesquels prime le jus ad bellum). Les droits reconnus aux individus par les nombreuses déclarations sont logiquement destinés, à un moment ou à un autre, à entrer en conflit avec l'idée même de souveraineté étatique. ${ }^{123}$

D'après Ferrajoli, Vitoria serait notamment à l'origine de l'idée de souveraineté étatique externe, "identifiée avec un ensemble de droits naturels des peuples idoines, d'un côté, à offrir une nouvelle légitimation de la conquête et, de l'autre, à apporter l'échafaudage idéologique du caractère eurocentrique du droit international, de ses attitudes colonisatrices voire même de ses vocations belliqueuses". 124

Vitoria démontre l'existence d'un grand nombre de droits naturels et universels qui, de fait, seuls les espagnols peuvent exercer. ${ }^{125}$ Parmi ces droits, à titre d'exemple, il suffira de citer le droit-devoir de correction

121 La conquête de l'Amérique (1982), Paris, Seuil, 1991, p. 487.

122 Pour une reconstruction précise des arguments de Francisco de Vitoria, nous renvoyons à Ferrajoli, L., "La sovranità nel mondo moderno. Crisi e metamorfosi”,, in M. Basciu, Crisi e metamorfosi della sovranità, Milano, Giuffrè, 1996, pp. 21 et ss., et, surtout, à Gómez Robledo, A., "Fundadores del derecho internacional", in Derecho, México, vol. 9, Colegio Nacional, 2001, pp. 421 et ss.

123 Sur le lien étroit entre politiques de l'immigration et perte de souveraineté des Etats, notamment européens, voir Marie, C.-V., "Le traitement de l'immigration en France: une police de l'étranger au service d'une politique d'opinion", in I. Simon-Barouh et V. de Rudder (eds.), Migrations internationales et relations ethniques. Recherche, politique et société, Paris, Harmattan, 1999, p. 11.

124 Ferrajoli, L., op. cit., note 122, pp. 29-30 (nous traduisons). Dans le même sens, voir Becerra Ramírez, M., "Una visión diferente de las fuentes del derecho internacional”, in M. Becerra Ramírez et K. Müller Uhlenbrock (coords.), La juridificacion de las relaciones internacionales. Un análisis plural, México, Universidad Nacional Autónoma de México, 2008, pp. 116 et ss.

125 Ibidem, p. 31. 
fraternelle des barbares ${ }^{126}$ ce qui fonde "en droit" non seulement la conquête mais aussi une pratique comme celle du requerimiento.

L'histoire des droits de l'homme connaît une étape clé avec la philosophie des Lumières. Le même Todorov ${ }^{127}$ met en exergue la fragilité des conceptions de l'individu et de l'Etat propres aux philosophies centrées sur l'idée d'universalisme et de droits de l'homme. Un moment clé dans l'édification de cette philosophie a été la révolution française, au regard La consécration de la conception de l'individu et de la nation consacrée par la Déclaration des Droits de l'Homme et du Citoyen de 1789. aurait engendré une difficulté majeure, aussi bien conceptuelle que pratique: l'impossibilité de penser la différence autrement que comme une menace pour la communauté politique.

Des études sur les travaux de l'Assemblée Nationale Française sous la Convention ont montré toute l'ambiguïté de l'universalisme qui portait, en son sein, le germe de l'exclusion. ${ }^{128}$ En 1793, lors des débats relatifs à l'adoption des premiers décrets sur les étrangers, on retrouve le ton du requerimiento. Selon les propres mots du porte-parole du Comité de sûreté générale, prononcés avant de présenter son projet sur les etrangers:

D'après les principes éternels de justice qui gouvernent un peuple libre et humain, vous avez annoncé à l'univers que vous étiez les amis et les alliés de tous les peuples... aujourd'hui vos principes sont les mêmes... nous chercherons notre défense dans les droits sacrés de la nature; ils seront légitimes car la liberté dirigera nos coups, et l'univers délivré en recueillera les fruits.

L'argumentaire se termine par une garantie: on ne confondra pas "l'homme égaré qui revient avec le conspirateur qui se masque ou l'é-

126 "Correction fraterna est de iure naturali, sicut et dilectio. Cum ergo omnes illi sint non solum in peccatis, sed extra statum solutis, ergo ad Christianos spectat corrigere et dirigere eos, immo videtur quod teneantur ad hoc", Francisco de Vitoria, De Indis recenter inventis relectio prior (1539), in De indis et de iure belli relectiones. Relectiones theologicae XII, édition due aux soins de E. Nys, New York, Oceana, 1964, sect. III, 9, p. 262, cité par L. Ferrajoli, op. cit., note 122, p. 31 (note 35).

127 Todorov, T., Nous et les autres: la réflexion française sur la diversité humaine, Paris, Seuil, 1992.

128 Wahnich, S., L'impossible citoyen: l'étranger dans le discours de la Révolution française, Paris, A. Michel, 1997. 
tranger paisible qui aime nos lois avec celui qui intriguant et hypocrite en parle avec respect pour les trahir avec succès". ${ }^{129}$ La distinction entre les bons et les mauvais étrangers semble alors être consubstantielle à l'esprit républicain. ${ }^{130}$

L'histoire plus récente semble confirmer une telle analyse. D'après certains auteurs c'est l'identité européenne qui pose problème: elle “occulterait les valeurs non européennes" et elle "se serait exclusivement construite autour de valeurs de l'Europe 'blanche' (tradition gréco-romaine, héritage chrétien, libéralisme du siècle des lumières, etc.)". ${ }^{131} \mathrm{Par}$ ailleurs, si la citoyenneté européenne ajoute peu au sentiment d'appartenance des ressortissants communautaires, elle se traduit néanmoins par un multiplicateur du sentiment d'exclusion pour les autres qui, aujourd'hui, non seulement ne sont pas "français" ou "allemands", mais sont aussi "extra-communautaires". Comme le souligne Véronique de Rudder, "le principe d'inhospitalité est désormais le fondement même de la "politique d'immigration" de l'Union européenne qui, d'ailleurs, se réclame officiellement du principe de "préférence communautaire". Les dispositifs communautaires "instaurent/restaurent une hiérarchie ethnico-raciale du monde et jusqu'au sein même de l'espace sociopolitique européen". ${ }^{132}$

129 Cité par S. Wahnich, ibidem, pp. 27 et 32.

130 Sur la genèse du "national républicanisme exclusif", De Rudder, V., "Politiques d'immigration" en Europe. Du principe d'hospitalité à la règle d'inhospitalité", VEI Enjeux, 125, 2001, pp. 29-30. Streiff-Fénart, J., "A propos d' assimilation' et de 'multiculturalisme': quelques réflexions sur les catégorisations de l'altérité dans les sociétés de l'immigration", in I. Simon-Barouh - V. de Rudder (eds.), op. cit., note 123, p. 350, insiste sur l'efficacité redoutable du "schème classificatoire répartissant la population immigrée en bons et mauvais".

131 Flauss, J.-F., "L'action de l'Union européenne dans le domaine de la lutte contre le racisme et la xénophobie", $R T D H, 2001$, p. 487. B. Melis, Negotiating Europe's Immigration Frontiers, Kluwer, The Hague, 2001, p. 3, après avoir constaté que depuis 1989 les facteurs raciaux sont devenus très importants dans les politiques d'immigration, se questionne sur la compatibilité de la politique de l'Union européenne avec "the best European and liberal tradition". Sa thèse, selon laquelle les règles adoptées sont directement ou indirectement racistes ou sexistes, se fonde sur le constat d'une tendance, accrue à la fin du vingtième siècle, à construire une forteresse Europe: une tendance à l'exclusion, notamment dans les règles sur le regroupement familial.

132 De Rudder, V. (ed.), op. cit., note 123, p. 32. Pour un regard moins pessimiste qui, toutefois, met en évidence la frilosité des politiques bruxelloises, Ph. de Bruycker, 
L'enseignement que l'on peut tirer de l'expérience révolutionnaire française, aboutissant à 'l'exclusion comme nécessité de la souveraineté nationale"133 et de celle, plus récente, européenne, est que la notion d'étranger tend à recevoir un traitement "spécifique" dans un contexte de terreur. La Terreur révolutionnaire a offert le contexte idéal pour la consécration de l'identité étranger = suspect. La terreur liée aux attentats terroristes, notamment depuis le 11 septembre 2001, tend à consacrer l'identité immigré musulman $=$ terroriste en puissance ${ }^{134}$ et à légitimer toute sorte de mesure.

En effet, les enjeux liés à la répression du terrorisme sont susceptibles de mettre en évidence les défaillances de l'Etat, dont les devoirs, d'après la doctrine internationaliste, ${ }^{135}$ comprennent aujourd'hui, sans

"L'émergence d'une politique européenne d'immigration", in id. (sdd.), L'émergence d'une politique européenne d'immigration, Bruxelles, Bruylant, 2002.

133 Wahnich, S., op. cit., note 123, p. 11.

134 Sur cet aspect, voir l'étude récente de Gonzáles, M., op. cit., note 17, s'attachant à reconstruire les contributions des différents gouvernements des Etats-Unis à la création de l'image de l'"ennemi": "la tesis 'el mundo es amenazante', una interpretación simplista y maniquea, siempre ayuda a legitimar un gobierno (sobre todo en país de ignorantes) y le da cohesión nacional, ¿se imaginan un país sin ella? Por ello... ha sido necesario crear un enemigo, construir un alter (existente o no)" (p. 55). Mais surtout: "¿qué mejor amenaza que el terrorismo? Un enemigo sin fronteras, indefinido, impreciso, encabezado por 'demonios'. Para el idealismo estadounidense, los terroristas no son percibidos como seres humanos, son un enemigo perfecto" (p. 94). El concept d'Autrui, semble central dans l'appréhension du terroriste. Comme le souligne. Portilla Gómez, J. M., "Repercusiones del combate al terrorismo en el derecho humanitario y los derechos humanos", in Méndez Silva, R. (coord.), Derecho internacional de los derechos humanos. Culturas y Sistemas Jurídicos Comparados, México, Universidad Nacional Autónoma de México, 2008, t. 2, p. 101, "el terrorista es siempre el otro".

135 L'actualité de la question est témoignée par le thème du 41ème colloque annuel de la Société française pour le droit international, dédié à La responsabilité de protéger (Nanterre, 7-9 juin 2007). Sur les questions, très techniques, relative aux types de protections dont peuvent bénéficier les individus (nationaux ou étrangers) victimes d'atteintes à leur droits fondamentaux, nous renvoyons à une étude récente qui a le mérite de poser la question, également, à l'égard des individus victimes des sanctions individuelles prononcées par le Comité des sanctions de l'ONU: Forlati, S., "Protection diplomatique, droits de l'homme et réclamations 'directes' devant la Cour internationale de justice. Quelques réflexions en marge de l'arrêt Congo/Uganda", Revue générale de droit international public, 111, 1, 2007, pp. 89-116; sur les sanctions qui nous intéressent, voir notamment p. 103. Pour une réflexion plus large sur la nouvelle conception de la souveraineté sous-tendue par l'idée de responsabilité de proteger, voir López-Jacoiste Díaz, E., "La responsabilidad de proteger: reflexiones sobre su fundamento y articulación", Anuario de Derecho 
aucun doute, celui de "protéger" les individus. L'intervention de l'Union Européenne en matière de sanctions économiques individuelles et la jurisprudence la concernant semblent sérieusement compromettre les valeurs sur lesquelles l'Europe des droits semble fonder sa légitimité. Or, c'est pour préserver une telle bonne réputation que l'on assiste à un sursaut de l'Etat de droit.

\section{CONCLUSION: VERS UN RETOUR À L'ETAT DE DROIT?}

Il n'est pas inutile de citer intégralement le paragraphe conclusif du rapport de la commission des questions juridiques et des droits de l'homme du Conseil de l'Europe:

Une lutte contre le terrorisme et la criminalité en général, rigoureuse mais correcte, ne peut que renforcer la crédibilité des institutions démocratiques et affaiblir ainsi que délégitimer ses ennemis. Comment peut-on justifier de lutter contre le terrorisme en maintenant depuis plus de six ans sur la liste noire - et en le privant ainsi de ses doits fondamentaux, après avoir détruit l'œuvre de sa vie - une personne âgée de 78 ans, alors même que les autorités de poursuite pénale de deux pays différents n'ont absolument rien découvert de répréhensible à son encontre? Tout cela au nom d'une décision prise par l'organisation qui proclame sa "foi dans les droits fondamentaux de l'homme, dans la dignité et la valeur de la personne humaine" et qui s'engage "à créer les conditions nécessaires au maintien de la justice et du respect des obligations nées des traités et autres sources du droit international"; une décision appliquée sans broncher par des Etats qui ne manquent pourtant jamais une occasion d'affirmer leur attachement inconditionnel aux valeurs du Conseil de l'Europe. ${ }^{136}$

En dépit d'une telle prise de conscience et des vœux ainsi exprimés, la question initiale reste sans réponse. Les individus qui, de par leur patronyme ou leur pratique religieuse, laissent naître la suspicion internationale d'un "lien avec les réseaux terroristes" disposent-ils, à l'heure actuelle, dans les Etats ayant inventé les droits de l'homme, d'une pro-

Internacional, 22, 2006, pp. 285-315. Cette analyse, pp. 310 et ss., a le mérite de montrer la fragilité de l'idée d'une obligation, pour la Communauté internationale, de protéger tant que les membres permanents du Conseil de sécurité disposent d'un droit de veto qui pourrait bloquer une intervention "protectrice".

136 Rapport de Monsieur Dick Marty, du 16 novembre 2007, doc. 11454. 
tection effective? La Convention européenne des droits de l'homme ne risque-t-elle pas d'être immolée sur l'autel de la lutte internationale contre le terrorisme?

Or, paradoxalement, la lutte contre le terrorisme, telle qu'elle est mise en œuvre, pourrait être interprétée comme une victoire inespérée pour les terroristes: personne avant, et notamment aucun acte terroriste, n'avait réussi dans l'entreprise visant à discréditer les démocraties occidentales construites autour du mythe des droits de l'homme. ${ }^{137}$ Le Conseil de Sécurité, sous l'impulsion des Etats-Unis et avec la complice coopération - mais il faudrait peut-être dire collaboration- européenne, semblerait avoir réussi. Ce n'est pas sans pertinence que certains auteurs ont dénoncé le retour à une "méthode législative primitive" et la mise en péril du rechstaat. ${ }^{138}$

De tels sombres constats pourraient, au moins en ce qui concerne l'Europe, laisser la place à une vision plus sereine, éclairée par le retour de l'Etat de droit. En effet, au moment d'écrire ces dernières lignes, l'Avocat général auprès de la Cour de Justice des Communautés Européennes vient de rendre ses conclusions sur l'affaire Kadi, jugée, le 21 septembre 2005, conjointement à l'affaire Yusuf et soulevant des questions juridiques identiques. Monsieur Poiares Maduro cite à bon escient Aharon Barak, ancien président de la Cour suprême de l'État Israël:139

137 L'accent sur cet aspect est mis par Bennoune, K., “"To respect and to ensure': reconciling international human rights obligations in a time of terror", American Society of International Law, Poceedings of the 97th Annual Meeting, 2003, pp. 23-24: "Terrorism has won a great victory... because some of the world's leading democracies have shown themselves willing to undermine the rule of law, both domestically and internationally, something which the despicable international terrorists could never have achieved by themselves". Il s'agit, d'après Méndez Silva, R., "Derivaciones del 11 de septiembre para el derecho internacional", in id. (coord.), Derecho y seguridad internacional. Memoria del Congreso Internacional de Culturas y Sistemas Jurídicos Comparados, México, Universidad Nacional Autónoma de México, 2005, p. 109, d'un exemple de cercle vicieux des fondamentalismes.

138 Andersson, T. et al., op. cit., note 18: "Blacklisting is, quite simply a primitive legislative method... when blacklisting is used against individuals, the Rechstaat requires a constitutional standard of legal security".

139 Cour suprême de l'État d'Israël, HCJ 769/02 (2006), Le Comité public contre la torture en Israël e.a. / Gouvernement de l'État d'Israël, points 61 et 62, cité dans les Conclusions de l'Avocat général, paragraphe 46. 
C'est lorsque les canons grondent que nous avons particulièrement besoin du droit... Toute lutte menée par l'Etat — que ce soit contre le terrorisme ou contre un autre ennemi- doit être conduite selon les règles et en application du droit. Il existe toujours une législation à laquelle l'Etat doit se conformer. Il n'existe aucun "trou noir"... La raison qui sous tend cette approche n'est pas seulement la conséquence pratique de la réalité politique et normative. Ses racines sont bien plus profondes. Elle est l'expression de la différence entre un État démocratique qui lutte pour sa survie et le combat de terroristes qui se soulèvent contre lui. L'Etat combat au nom du droit et du respect du droit. Les terroristes combattent le droit en le violant. La guerre contre le terrorisme est également une guerre du droit contre ceux qui le combattent.

Ayant à l'esprit un tel enseignement, l'Avocat général non seulement propose à sa cour d'annuler, à la fois, l'arrêt du tribunal de première instance et le règlement communautaire reproduisant la liste noire (la même sur laquelle figure Monsieur Yusuf), dans la mesure où elle concerne Monsieur Kadi, mais rappelle aussi avec insistance l'attachement de la Communauté Européenne au respect des droits de l'homme. D'après celui-ci, "le Tribunal a commis une erreur de droit en jugeant qu'il n'était pas compétent pour contrôler le règlement attaqué à la lumière des droits fondamentaux qui font partie des principes généraux du droit". ${ }^{140}$ Par ailleurs, il revient sur les rapports entre Communauté et ONU:

la Cour ne saurait... faire fi des valeurs fondamentales sur lesquelles repose l'ordre juridique communautaire et qu'elle se doit de protéger. Le respect des autres institutions n'a de sens que s'il repose sur une compréhension partagée de ces valeurs et sur un engagement mutuel à les protéger. Par conséquent, dans des situations dans lesquelles des valeurs fondamentales de la Communauté sont en jeu, la Cour peut être appelée à apprécier, et éventuellement à annuler, des mesures adoptées par les institutions communautaires, même lorsque ces mesures reflètent les souhaits du Conseil de sécurité. ${ }^{141}$

L'argument de la sécurité ne peut donc fonctionner comme un blanc seing car "le processus politique risque d'être trop réactif face à l'urgence des préoccupations populaires, amenant les autorités à dissiper les 
craintes du plus grand nombre au détriment des droits de quelques uns". ${ }^{142}$ Alors même que la juge communautaire ne dispose pas des éléments nécessaires pour juger du caractère injustifié et/ou disproportionnés des sanctions dont il s'agit, "la simple possibilité qu'il puisse en être ainsi constitue un anathème dans une société qui respecte la prééminence du droit". ${ }^{143}$

On ne peut que se réjouir d'un tel sursaut qui semble mettre en avant les exigences les plus élémentaires d'un Etat de droit: encore faut-il que la Cour de justice suive les conclusions de son Avocat général ce qui, compte tenu du contexte politique, est loin d'aller de soi. 